\title{
Improved method for subsystems performance trade-off in system-of-systems oriented design of UAV swarms
}

\author{
ZHANG Ruiwen, SONG Bifeng, PEI Yang*, and YUN Qijia \\ School of Aeronautics, Northwestern Polytechnical University, Xi' an 710072, China
}

\begin{abstract}
For the rapidly developing unmanned aerial vehicle (UAV) swarm, the system-of-systems (SoS) oriented design is a prospective conceptual design methodology due to the competence for complex mission requirements and subsystems interactions. In the SoS oriented design, the subsystems performance trade-off is the basis of design decisions. In the trade-off for surveillance missions, most previous works do not consider track reporting and mainly focus on the design of platforms. An improved method for the subsystems performance trade-off in the SoS oriented UAV swarm design is proposed. Within an improved design framework with subsystems disaggregation, this method is characterized by treating platforms, sensors, and communications as equally important subsystems, integrating operational strategies into the trade-off, and enabling the trade-off for track reporting. Those advantages are achieved by a behavior-based modular model structure for agent-based operational modeling and simulation. In addition, a method of analyzing the bounds of the communication range is also presented. Simulation experiments are conducted by using precision-based simulation replication rules and surrogate modeling methods. The results demonstrate the effectiveness of the proposed method, and show that the configuration of area partitioning changes the trade space of subsystems performances, indicating the necessity of integrating operational strategies into the conceptual design.
\end{abstract}

Keywords: swarm, system-of-systems (SoS), trade-off, agent, area partitioning.

DOI: $10.21629 /$ JSEE.2019.04.10

\section{Introduction}

In recent years, unmanned aerial vehicle (UAV) swarms have attracted increasing attention due to the advantages of robustness, flexibility, and scalability over the single UAV system in various missions [1]. In swarming operations, the weapon system is the whole swarm rather than any single UAV. That transition is a challenging traditional

\footnotetext{
Manuscript received August 13, 2018.

*Corresponding author.

This work was supported by the National Natural Science Foundation of China (11872314), and the National Key Research and Development Program of China (2017YFB1300102).
}

single-aircraft oriented conceptual design with more complex mission requirements and subsystems interactions. The system-of-systems ( $\mathrm{SoS}$ ) oriented design is a new methodology proposed recently [2,3], which is characterized by emphasizing the mission capability or effectiveness of the SoS rather than the performance of the single aircraft. Enabled by design frameworks directly connecting mission requirements with design analysis and other elements at the same time, the SoS oriented design produces better solutions for complex mission requirements and subsystems interactions, showing the prospect for the UAV swarm design.

Surveillance is a typical type of missions for UAV swarms, and the SoS oriented UAV swarm design for surveillance missions is extensively studied. A representative design framework is the two-level framework [4], containing a higher-level loop to evaluate the mission effectiveness of design alternatives and a lower-level loop to optimize the vehicle sizing. Durand et al. [1] further improved the framework by replacing the sequential optimization with global optimization and applied it to the multi-robot system design for mapping and exploration. Similarly, Tian et al. [5] proposed a combination of parameters, objective analysis, operational simulation, keyparameters analysis (COOK) framework of the SoS oriented design space exploration for UAV-helicopter groups, which contains four steps: combination of parameters, object analysis, operational simulation, and key-parameter analysis.

Subsystems performance trade-off provides the basis of design decisions in the SoS oriented design. The agentbased operational modelling and simulation (M\&S) is currently the most prevailing method, because it involves the uncertainty of mission scenarios as well as the dynamics of subsystems and is more feasible and adaptable due to the bottom-to-up fashion. Ranque et al. [6] used the agentbased operational M\&S in the trade-off of subsystems performances to support the design of commercial aircraft 
families for maritime monitoring, studying the trade-off among the number of aircraft, velocity, fuel mass, and radar range, et al. Similar works include the trade-off between velocity and radar cross section (RCS) for penetration missions [7], as well as the trade-off among velocity, lift-drag ratio, and detection probability for multi-aircraft search and rescue missions [8].

However, in most previous works, the missions are merely area coverage [1], target detection $[4,6,8]$, or target identification [9], while the real-time target track reporting is seldom addressed. Tracks are more representative to measure the quality of surveillance information, and also essential to post-surveillance operations such as defence and attack. Therefore the deficiency impedes more comprehensive trade-offs for surveillance missions.

In addition, most previous works mainly focus on the design of platforms, while sensors and communications are treated as subordinates $[1,4,6,8,9]$. That perspective is inapplicable to the SoS oriented UAV swarm design. First, the pursuit of flexibility and scalability requires sensors not to be integrated parts but to be modular suites that can be altered with missions and upgraded over time. Second, swarming operations are highly dependent on communication networks. Therefore, it is required to disaggregate sensors and communications from platforms, treating them as composing subsystems with equal status [10].

A critical cause of the above deficiencies lies in the operational model structure. Most previous works use simplex and monolithic model structures in the agent-based operational M\&S [1,4,6-9]. Those structures do not disaggregate the behaviors of platforms, sensors, and communications, thus are difficult to model the complex process of track reporting which involves the interactions of those behaviors. Li et al. [11] proposed a modular modeling method for the operational M\&S, which created modular models of different subsystems and composed them according to the needs of different applications. That method is applicable to the concept of subsystems disaggregation, showing prospects to improve the above deficiencies.

Besides subsystems, the SoS architectures enable the operation of the SoS, which should be considered in the SoS oriented design. Issues of the architecture design include the system organization, network topology, command and control (C2) structures, and operational strategies, etc. Giles et al. [12] studied the top-to-down missionbased architecture for UAV swarms. Shu et al. $[13,14]$ studied the optimization of the SoS architecture, which included missions, equipment, and $\mathrm{C} 2$ structures. $\mathrm{Li}$ et al. $[15,16]$ proposed a formal information representation method of the system organization, and studied the optimization of the system organization for higher teamwork efficiency and more balanced task allocation. Tran et al. [9] studied the trade-off of $\mathrm{C} 2$ network topologies for situational awareness. Aksaray et al. [17] studied message passing and connectivity maintenance strategies in multi-UAV surveillance. In surveillance missions, operational strategies mainly include surveillance methods and the number of UAVs to deploy. Previous works seldom consider operational strategies as part of trade-offs to analyze their effects on subsystems performances, and pervious modular model structures also make it difficult to do that, because the decomposition is based on physical components rather than behaviors and could not be used to model and analyze the physical design and operational strategies separately.

Therefore, following the framework of the SoS oriented design, this paper proposes an improved method of the subsystems performance trade-off for surveillance UAV swarms. First, an improved design framework with subsystems disaggregation is presented, in which platforms, sensors, and communications are treated as equal-status subsystems. Within the framework, a behavior-based modular model structure for the agent-based operational M\&S is proposed, which is aimed at disaggregating subsystems, integrating operational strategies and modeling track reporting. To improve the efficiency of the analysis, a method of analyzing the bounds of the communication range is also presented. Finally, simulation experiments are conducted to demonstrate the proposed method.

\section{Problem formulation and assumptions}

\subsection{Typical SoS oriented design framework}

A typical two-level SoS oriented design framework is shown in Fig. $1[1,4]$. The swarm level evaluates the mission effectiveness of design alternatives by the operational $\mathrm{M} \& \mathrm{~S}$, and the aircraft level produces design alternatives according to given requirements.

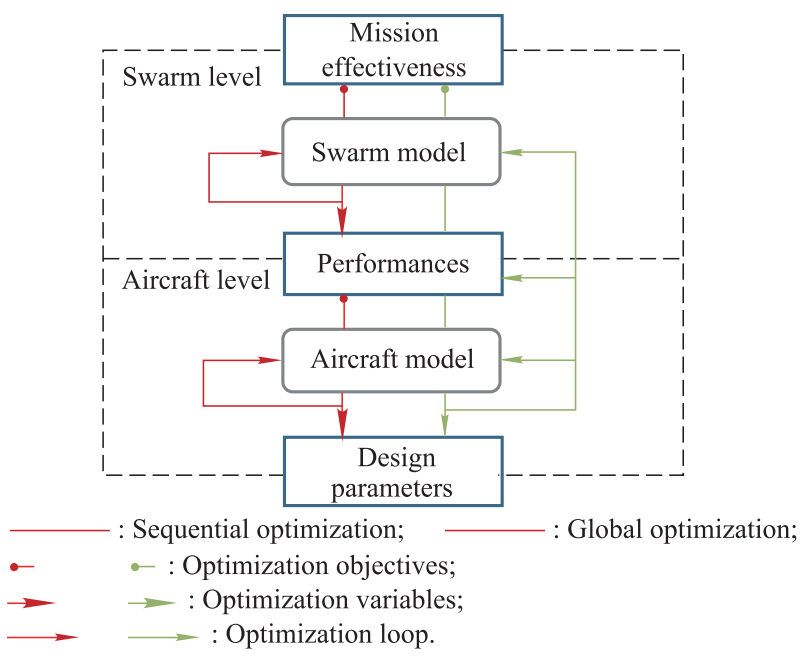

Fig. 1 Typical SoS oriented design framework 
The global optimization directly outputs design parameters with global optimality, but it requires detailed models. Therefore the sequential optimization is more prevailing currently in engineering as it has separate loops in each level. Subsystems performance trade-offs are essential in the sequential optimization, while provide references in the global optimization.

\subsection{Mission scenario}

We consider the scenario where a UAV swarm is deployed in the ground area surveillance for a given duration, reporting the tracks of moving ground vehicles (abbreviated as vehicles below) in the area to the commander. Vehicles are assumed to be non-confrontational. The commander is assumed to be a stationary control station.

A track is a set of associated state estimates describing the time-sequenced location, velocity, acceleration, and other characteristics of mobile objects [18]. We only consider position and velocity estimates. The creation and transmission of other information are also ignored. The state of an object $i$ at the time $t$ is defined as

$$
\boldsymbol{X}_{i}(t)=\left[x_{i}(t), x_{i}^{\prime}(t), y_{i}(t), y_{i}^{\prime}(t), z_{i}(t), z_{i}^{\prime}(t)\right]
$$

where $x_{i}(t), y_{i}(t)$, and $z_{i}(t)$ are coordinates in the Cartesian space; $x_{i}^{\prime}(t), y_{i}^{\prime}(t)$, and $z_{i}^{\prime}(t)$ are velocity components in $x, y$, and $z$ coordinates respectively. The state estimate of the object is defined as

$$
\widehat{\boldsymbol{X}}_{i}(t)=\left[\widehat{x}_{i}(t), \widehat{x}_{i}^{\prime}(t), \widehat{y}_{i}(t), \widehat{y}_{i}^{\prime}(t), \widehat{z}_{i}(t), \widehat{z}_{i}^{\prime}(t)\right]
$$

where $\widehat{\cdot}$ denotes the estimate of the corresponding component in (1). The ground is assumed to be flat at the sea level. Thus $z$ components in states and state estimates of vehicles remain zero.

The demonstration of the mission scenario is shown in Fig. 2. Track reporting works as follows. Each UAV detects the targets in the neighboring area, generates tracks, and sends to the commander. The commander fuses the received local tracks into global tracks, creating the single integrated situational awareness.

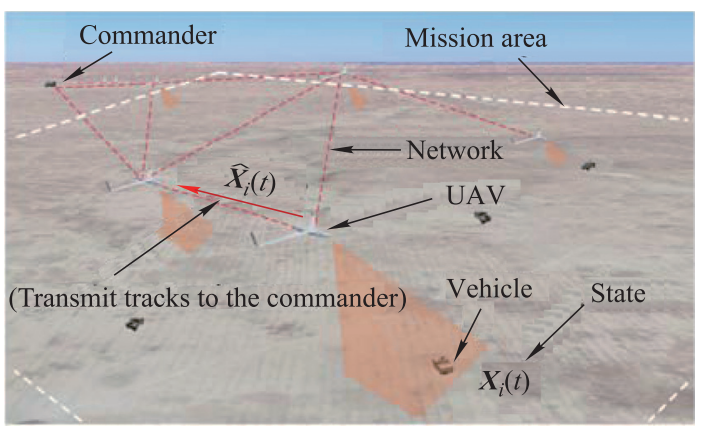

Fig. 2 Demonstration of the mission scenario
Tracks are processed and transmitted periodically and asynchronously among agents. The transmission period is longer than the processing period, and only the most updated tracks in each transmission period are transmitted.

\subsection{Measures of effectiveness}

We use two measures of effectiveness (MoEs) to quantify the information quality, i.e., completeness, timeliness, and the accuracy of global integrated situation awareness. The overall tracking ratio $r_{\text {tro }}$ is the quantification of completeness. The overall tracking error $e_{\text {tro }}$ is the comprehensive quantification of accuracy and timeliness, because the global tracking error is induced from both local tracking and transmission delays.

The overall tracking ratio is defined as

$$
r_{\text {tro }}=\frac{1}{T_{\mathrm{m}}} \int_{0}^{T_{\mathrm{m}}} \frac{n_{\mathrm{tr}, \mathrm{c}}(t)}{n_{\mathrm{tg}}(t)} \mathrm{d} t
$$

where $n_{\mathrm{tr}, \mathrm{c}}(t)$ is the number of tracks recorded by the commander at the time $t, n_{\mathrm{tg}}(t)$ is the number of targets at the time $t$, and $T_{\mathrm{m}}$ is the duration of the mission. We only consider the error of position estimates. The tracking error of a target $i$ that an agent $j$ records at the time $t$ is defined as

$$
e_{\mathrm{tr}, i, j}(t)=\sqrt{\left(\widehat{x}_{i, j}(t)-x_{i}(t)\right)^{2}+\left(\widehat{y}_{i, j}(t)-y_{i}(t)\right)^{2}}
$$

where the subscript $j$ means the estimate is produced by the agent $j$. Then the overall tracking error is defined as

$$
e_{\text {tro }}=\frac{1}{T_{\mathrm{m}}} \int_{0}^{T_{\mathrm{m}}} \frac{1}{n_{\mathrm{tr}, \mathrm{c}}(t)} \sum_{i=1}^{n_{\mathrm{tr}, \mathrm{c}}(t)} e_{\mathrm{tr}, i, \mathrm{c}}(t) \mathrm{d} t
$$

where $e_{\mathrm{tr}, i, \mathrm{c}}(t)$ is the tracking error of the target $i$ recorded by the commander at the time $t$. The integrals are calculated by numerical methods, in which sampling steps synchronize with the processing steps of the commander.

\subsection{Performances of interest}

We consider the small tactical UAV [10] swarm equipped with electro-optical/infrared (EO/IR) sensors and multihop ad hoc communication networks. The platform performances mainly include cruise velocity, cruise altitude, and endurance. We assume that UAVs fly at the constant cruise velocity and altitude for the maximum endurance, and mainly consider cruise velocity $v_{\mathrm{a}}$ in the trade-off, as the endurance is constrained by the mission, and the flight altitude is usually specified by regulations.

Sensor performances mainly include the field of view (FOV), detection probability, and tracking error. The FOV and detection probability depend on imaging elements which are usually off-the-shelf products. By using the Cramér-Rao lower bound (CRLB) in analysis, we ignore the dependence of tracking errors on filtering algorithms, 
and only consider sensor measurement errors in the tradeoff. The measurement errors of the target $i$ at the time $t$ converted to the Cartesian space are expressed as follows:

$$
\begin{aligned}
& e_{\mathrm{m}, x}(t)=\widetilde{x}_{i}(t)-x_{i}(t) \\
& e_{\mathrm{m}, y}(t)=\widetilde{y}_{i}(t)-y_{i}(t)
\end{aligned}
$$

where $\widetilde{x}_{i}(t)$ and $\widetilde{y}_{i}(t)$ are converted measurements in Cartesian coordinates. We assume the measurement errors in both $x$ and $y$ coordinates follow the normal distribution, of which the means are zero and the variances $\sigma_{\mathrm{m}, x}$ and $\sigma_{\mathrm{m}, y}$ are identical constants. Then the synthetic measurement error, expressed as

$$
e_{\mathrm{m}}(t)=\sqrt{e_{\mathrm{m}, x}(t)^{2}+e_{\mathrm{m}, y}(t)^{2}},
$$

follows the Rayleigh distribution with the mean

$$
\sigma_{\mathrm{m}}=\sqrt{\frac{\pi}{2}} \sigma_{\mathrm{m}, x}=\sqrt{\frac{\pi}{2}} \sigma_{\mathrm{m}, y}
$$

where $\sigma_{\mathrm{m}}$ is used as the metric of measurement errors.

Communication performances mainly include the communication range, delay, and loss rates. We only consider the communication range $r_{\mathrm{c}}$ and the single-hop delay $t_{\mathrm{d}}$ in the trade-off as loss rates are usually low, assuming that $r_{\mathrm{c}}$ and $t_{\mathrm{d}}$ are constants and messages can be transmitted bidirectionally without loss when agents are within the communication range.

\section{Proposed approach}

\subsection{Improved SoS oriented design framework}

With the concept of subsystems disaggregation, we propose an improved design framework, as shown in Fig. 3. In this framework, the aircraft level is disaggregated into three parts based on subsystems. Performances, models, and design parameters are disaggregated accordingly. The three subsystems are modeled and optimized separately, and the reciprocal constraints among them are considered.

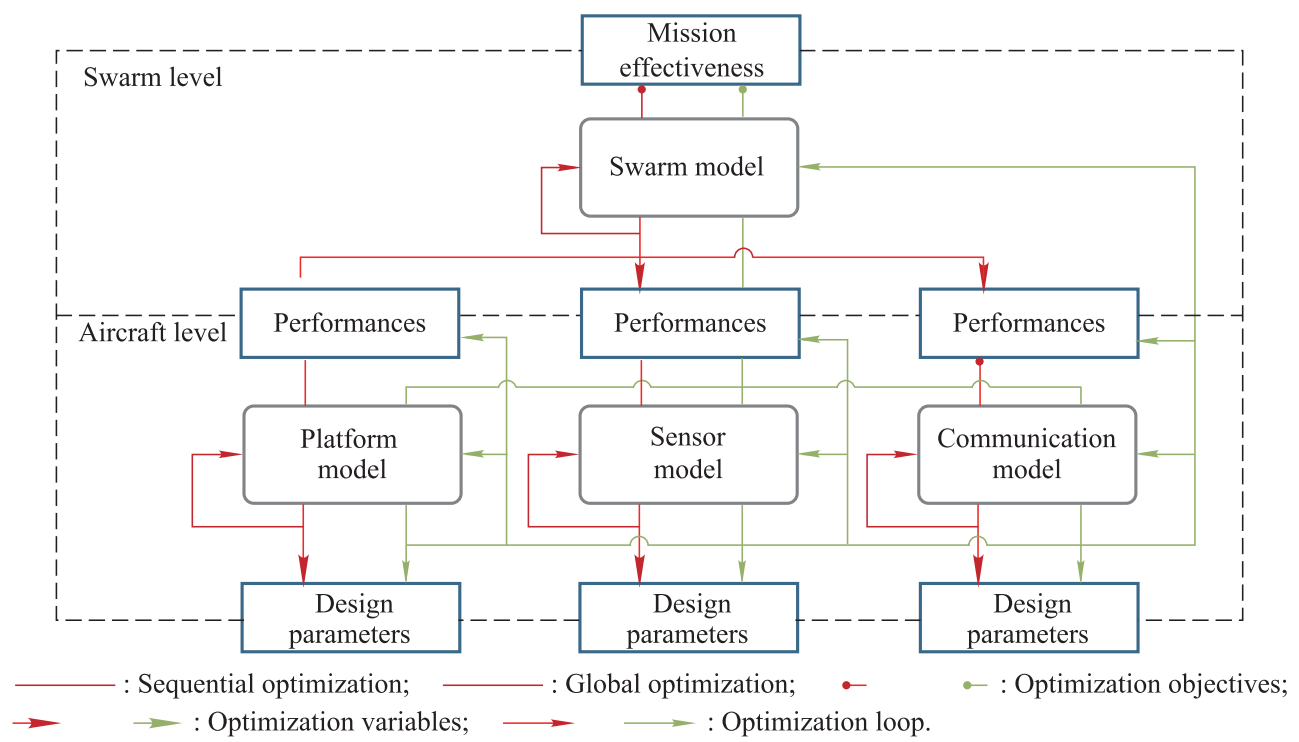

Fig. 3 Improved SoS oriented design framework

\subsection{Behavior-based modular model structure}

The behavior-based modular model structure for the agentbased operational M\&S is shown in Fig. 4 in the form of the unified modeling language (UML) class diagram. For the mission scenario studied in this paper, there are three types of entities: aircraft, commander and vehicle. Each entity is treated as an agent and modeled as an instance of a corresponding class. Previous works either use simplex and monolithic structures to model agent behaviors in single classes, or use modular structures and decompose based on physical components such as sensors, platforms and communications. In the proposed model structure, agent classes are decomposed into multiple modular element classes based on behaviors. High-level behaviors related to operational strategies are separated from basic behaviors related to the physical design. Therefore, subsystems and operational strategies can be modelled and analyzed separately.

In the model, entity and element are base classes. The aircraft, commander, and vehicle are the generalizations of the entity, while the platform, sensor, communication terminal (CmcTmn), situation manager (SituMngr), mission manager (MsnMngr), and central decision maker (CenDecMkr) are the generalizations of the element. Each instance of the entity is an agent and a composite of element instances. 


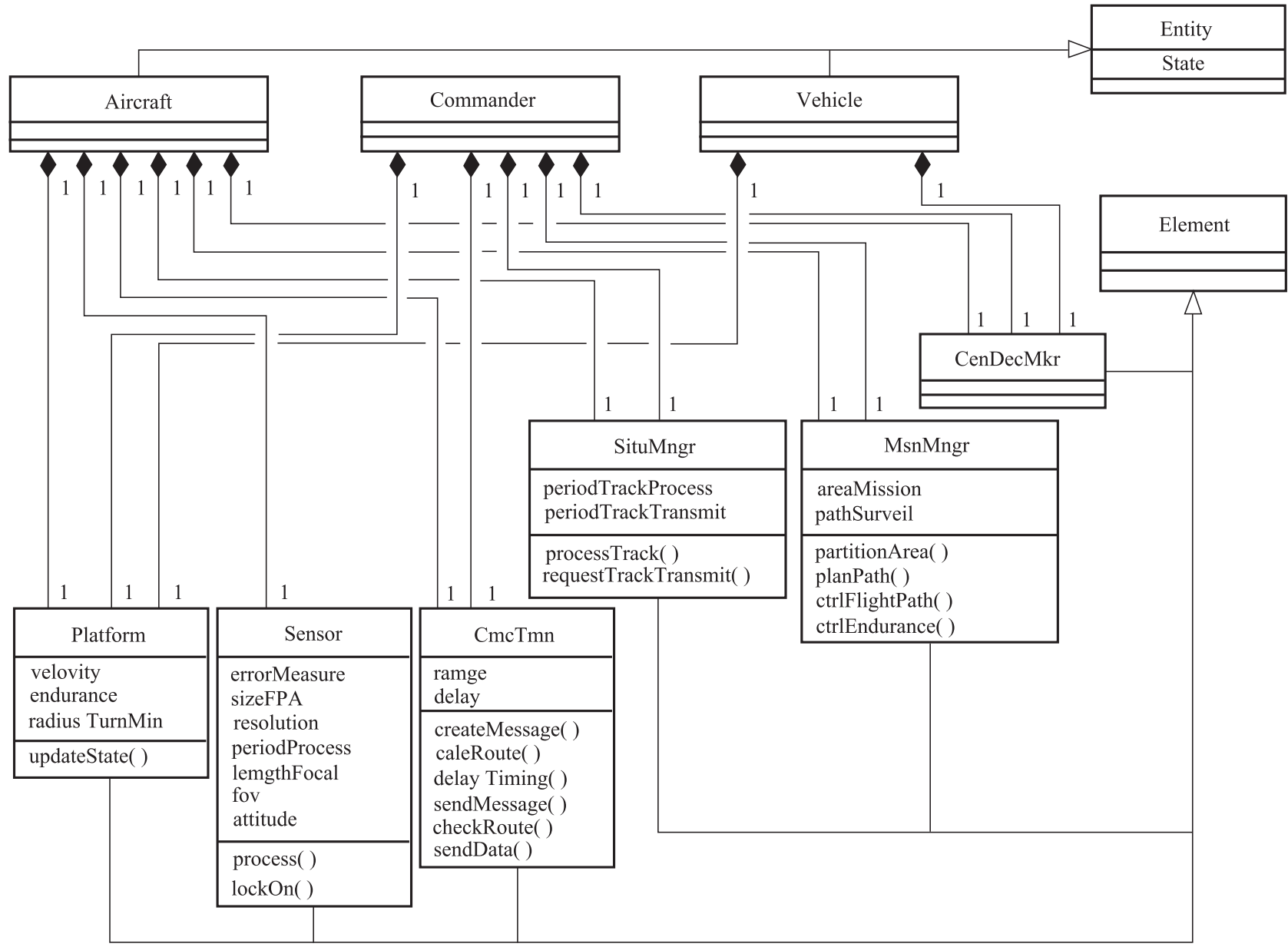

Fig. 4 Behavior-based modular model structure

Generalizations of the element are divided into three levels. The platform, sensor and CmcTmn are bottom-level element generalizations. The platform performs movement behaviors. The state is updated by three degree-of-freedom kinetic equations and controlled by overloads, as described in [19]. The sensor performs target detection and track generation behaviors, with the capability of changing the focal length and attitude to lock on targets. The CmcTmn performs message sending and receiving behaviors.

The SituMngr and the MsnMngr are medium-level element generalizations. The SituMngr performs track management behaviors, including track fusion and track transmission control. The MsnMngr performs mission management behaviors, including area partitioning, surveillance path planning, path-flight control, and endurance control.

The CenDecMkr is the top-level element generalizations. It performs high-level decision making behaviors, including mission stage control (entering and withdraw), emergency response, etc.

The variation of entity generalizations is realized by the variation of composition. The entity only aggregates necessary element generalizations according to the expected behaviors, such as the commander not aggregating the sensor and the vehicle only aggregating the platform.

The proposed model structure is scalable and extendable. Together with the improved design framework, it can also be used to study other issues of the SoS architecture by adding corresponding medium-level element generalizations. For example, to study the system organization, a system organization manager class can be added to manage organization behaviors. To study the design of the network topology, a network manager class can be added to manage the topology and message transmission.

\subsection{Modeling of track reporting}

For the aircraft, at each processing step, the sensor calculates the detection probability of targets in the field of view and generates tracks following the $m / n$ logic sliding windows criteria [20]. Then the SituMngr updates track records, assesses threat, and controls the sensor to lock on the largest-threat track. When the transmission step comes, 
the SituMngr requests the CmcTmn to send the most updated tracks in the previous transmission period. For the commander, at each processing step, the SituMngr fuses received tracks and updates track records. Received tracks are kept for two transmission periods. Detailed models are presented below.

\section{(i) Target detection}

The geometry of the EO/IR sensor detection is shown in Fig. 5, where $h_{\mathrm{a}}$ is the flight altitude, $\vartheta_{\mathrm{h}}$ and $\vartheta_{\mathrm{v}}$ are horizontal and vertical FOV respectively, $\delta_{\mathrm{n}}$ is the look-down angle, $x_{\mathrm{b}}$ is the longitudinal axis of the platform, and $x_{\mathrm{s}}$ is the normal axis of the FPA. The FOV is given by [21]

$$
\begin{aligned}
& \vartheta_{\mathrm{h}}=2 \arctan \left(\frac{h_{\mathrm{FPA}}}{2 f_{\mathrm{c}}}\right) \\
& \vartheta_{\mathrm{v}}=2 \arctan \left(\frac{w_{\mathrm{FPA}}}{2 f_{\mathrm{c}}}\right)
\end{aligned}
$$

where $h_{\mathrm{FPA}}$ and $w_{\mathrm{FPA}}$ are the height and width of the FPA respectively, and $f_{\mathrm{c}}$ is the focal length. The bottom width of the sensor footprint $w_{\mathrm{s}}$ is treated as the sweep width for surveillance path planning.

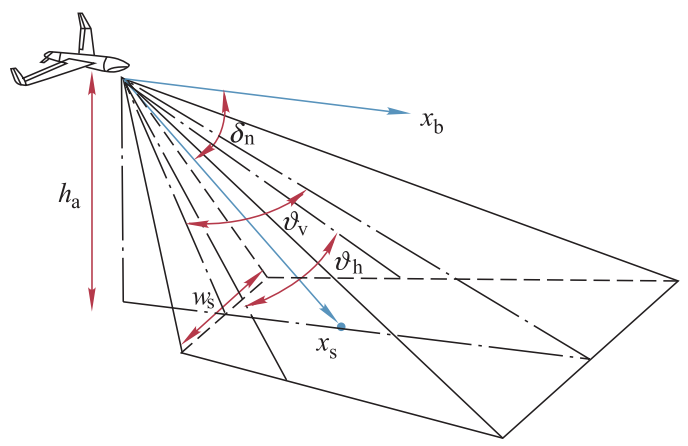

Fig. 5 Geometry of sensor detection

The probability of discrimination, including detection, recognition and identification, is given by the Johnson criteria [21]. The probability is determined by the dimension and relative position of targets, the resolution of the FPA, focal length, and FOV. The resolution of the FPA is expressed as the number of horizonal pixels $P i x_{\mathrm{h}}$ and vertical pixels $P i x_{\mathrm{v}}$. Details are described in [21].

When a track is locked on, $x_{\mathrm{s}}$ will point to the track position, and $f_{\mathrm{c}}$ will be changed to maintain a given projected size of the target on the FPA. Otherwise, $x_{\mathrm{s}}$ will point downwards in the symmetry plane of the platform with a default $\delta_{\mathrm{n}}$, and $f_{\mathrm{c}}$ will remain the lower limit.

(ii) Track generation

The state estimates are calculated by filtering algorithms recursively from motion models and measurement models, expressed as follows [20]:

$$
\begin{gathered}
\boldsymbol{X}_{i}\left(t_{k+1}\right)=\boldsymbol{f}_{(k)}\left(\boldsymbol{X}_{i}\left(t_{k}\right)\right)+\boldsymbol{G}_{(k)} \boldsymbol{v}_{(k)} \\
\boldsymbol{Z}_{i}\left(t_{k}\right)=\boldsymbol{h}_{(k)}\left(\boldsymbol{X}_{i}\left(t_{k}\right)\right)+\boldsymbol{W}_{(k)}
\end{gathered}
$$

where $k$ denotes the processing step, $t_{k}$ is the time at the step $k, \boldsymbol{f}_{(k)}(\cdot)$ is the state transition function, $\boldsymbol{v}_{(k)}$ is the process noise following the zero-mean Gaussian distribution with the covariance $\boldsymbol{Q}_{(k)}, \boldsymbol{G}_{(k)}$ is the process noise distribution matrix, $\boldsymbol{Z}_{(k)}$ is the sensor measurement, $\boldsymbol{h}_{(k)}(\cdot)$ is the measurement function, and $\boldsymbol{W}_{(k)}$ is the measurement error following the zero-mean Gaussian distribution with the covariance $\boldsymbol{R}_{(k)}$. The nearly constant velocity motion model with the discrete white noise acceleration and the converted measurement model are used, which are described in [22]. $\boldsymbol{R}_{(k)}$ is expressed as follows:

$$
\boldsymbol{R}_{(k)}=\left[\begin{array}{cc}
\sigma_{\mathrm{m}, x}^{2} & 0 \\
0 & \sigma_{\mathrm{m}, y}^{2}
\end{array}\right] .
$$

Regardless of filtering algorithms, the covariance of estimates cannot exceed a lower limit, called CRLB, is expressed as follows [23]:

$$
\begin{gathered}
\boldsymbol{P}_{i}\left(t_{k}\right)=\mathrm{E}\left[\left(\widehat{\boldsymbol{X}}_{i}\left(t_{k}\right)-\boldsymbol{X}_{i}\left(t_{k}\right)\right)\left(\widehat{\boldsymbol{X}}_{i}\left(t_{k}\right)-\boldsymbol{X}_{i}\left(t_{k}\right)\right)^{\mathrm{T}}\right] \geqslant \\
\boldsymbol{J}_{i}\left(t_{k}\right)^{-1}
\end{gathered}
$$

where $\boldsymbol{J}_{i}\left(t_{k}\right)$ is the fisher information matrix. $\boldsymbol{J}_{i}\left(t_{k}\right)$ can be calculated recursively, which is described in [20,23]. Then, the estimate $\widehat{\boldsymbol{X}}_{i}\left(t_{k}\right)$ is created by using the normal distribution, which is expressed as follows:

$$
\widehat{\boldsymbol{X}}_{i}\left(t_{k}\right) \sim N\left(\boldsymbol{X}_{i}\left(t_{k}\right), \boldsymbol{J}_{i}\left(t_{k}\right)^{-1}\right)
$$

(iii) Track synchronization and fusion

Track synchronization is calculated by the extrapolation method, and the track fusion is calculated by the suboptimal distributed track fusion method, which is described in [20].

(iv) Communication

As assumed in Section 2.4, the network is constructed based on the communication range. The message transmission process is shown in Fig. 6. When receiving a sending request from the element, such as sending tracks from the SituMngr, the CmcTmn creates a corresponding message and calculates a multi-hop route by the Dijkstra algorithm to the destination. If such a route exists, the CmcTmn writes the routing information in the message and sends out after the delay. Otherwise, the message will be discarded. When receiving a message, the $\mathrm{CmcTmn}$ first checks the routing information. If it is the destination, the data in the message will be sent to the corresponding element, such as to the SituMngr if the data is a track. Otherwise, the message will join the sending process and continue transmission. 


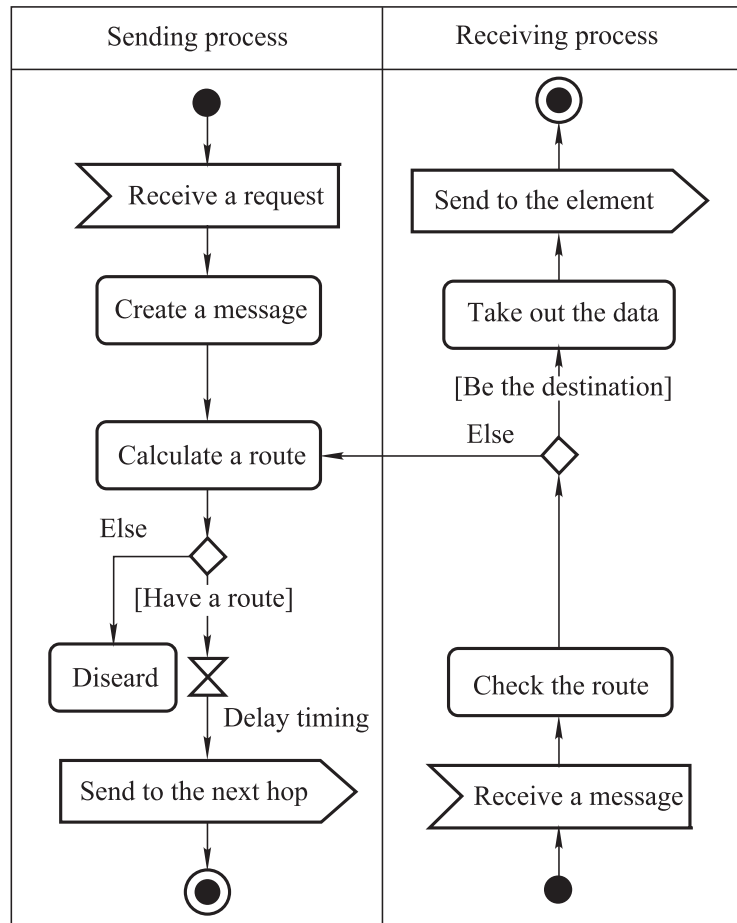

Fig. 6 Process of communication

\subsection{Surveillance method}

For surveillance missions, operational strategies mainly include surveillance methods and the number of UAVs to deploy $n_{\mathrm{a}}$. Currently, there are two types of surveillance methods: the sweep methods and the self-organized methods. In the sweep methods, a mission area is divided into multiple partitions, and each UAV surveils one partition in the sweep motion [24]. In the self-organized methods, UAVs plan paths dynamically and coordinate the paths by self-organization mechanisms [25]. Currently, the sweep methods are more technically mature and prevailing in engineering.

The area partitioning and path planning algorithms are described in $[24,26]$. We mainly consider the configuration of area partitioning. The vector configuration and the grid configuration are two typical configurations [24], as shown in Fig. 7. In both configurations, different partitions have an equal area. The vector configuration maximizes the length-width ratio of partitions, so as to extend the straight level flight. The grid configuration minimizes the lengthwidth ratio of partitions to make it close to 1 , pursuing the uniform distribution of UAVs. The width of partitions is larger than the sweeping width $w_{\mathrm{s}}$, and avoids excessive sensor footprint overlaps and turn-around flight.

Area partitioning and path planning are calculated before the mission and recorded in the MsnMngr. The flight along waypoints is controlled by the MsnMngr in the way of changing overloads in the platform.

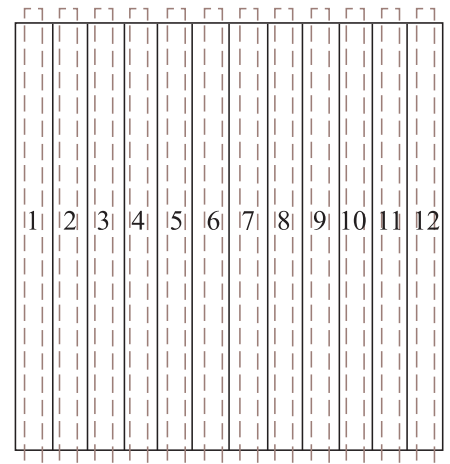

(a) Vector configuration

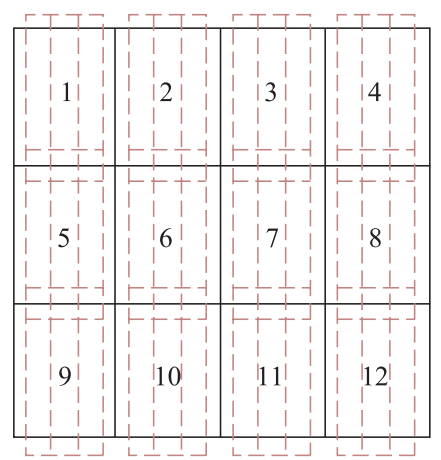

(b) Grid configuration

Fig. 7 Configuration of area partitioning

\subsection{Bounds of communication range}

The bounds of subsystems performances in the trade-off depend on both mission requirements and design candidates. We propose a method to determine the missionrelated bounds of communication ranges. Other bounds are determined by the engineering experience.

The upper bound of communication range $r_{\mathrm{c}, \mathrm{ub}}$ is the range to cover the entire mission area centered at the commander, making all UAVs directly communicating with the commander. But such a long range is seldom necessary when multi-hop links are constructed. Therefore, the lower bound of the communication range $r_{\mathrm{c}, \mathrm{lb}}$ is the minimum range to construct such links for all UAVs throughout the mission. The relative neighborhood graph (RNG) can be used to solve the problem.

The RNG is defined as a graph only containing edges satisfying the following conditions [27]:

An edge $(u, v)$ exists between vertices $u$ and $v$ if the distance between them $d(u, v)$ is less than or equal to the distance between every other vertex $w$, and whichever of $u$ and $v$ is farther from $w$, which is expressed as follows:

$$
\forall w \neq u, v: d(u, v) \leqslant \max [d(u, w), d(w, v)] .
$$

The demonstration of the RNG is shown in Fig. 8. The minimum communication range required at a certain mo- 
ment is the longest edge in the RNG of the communication network at that moment, ensuring that all agents connect to each other. The length of edges corresponds to the distance between UAVs, and the maximum distance occurs when two UAVs are at the corners of their area partitions; thus $r_{\mathrm{c}, \mathrm{lb}}$ is the maximum of all possible cases above. That depends on the size of the mission area, the number of UAVs $n_{\mathrm{a}}$, and the partitioning configuration, so it can be calculated before simulation experiments.

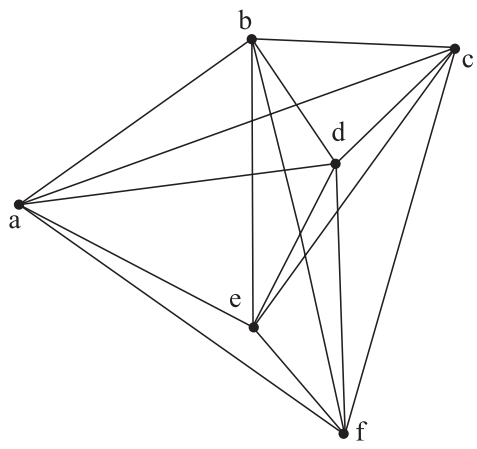

(a) Original graph

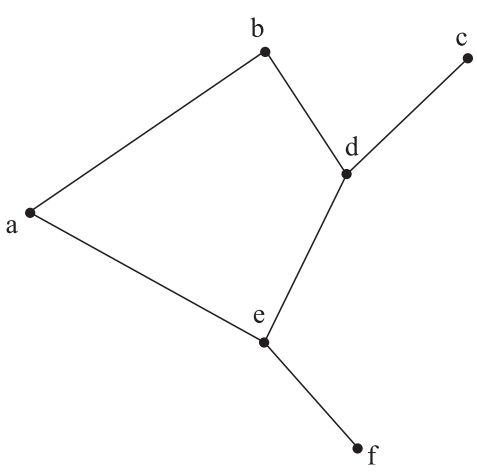

(b) RNG

Fig. 8 Demonstration of RNG

The number of possible cases is $4^{n_{\mathrm{a}}}$, which becomes innumerous for the exhaustive search when $n_{\mathrm{a}}$ is large. As a solution with the lower accuracy is already acceptable for the trade-off, the Monte Carlo method is used to solve it.

\section{Simulation experiments}

\subsection{Experiment settings}

We simplify the mission scenario described in Section 2.2 to the simulation case below. The mission area is a $12 \mathrm{~km} \times$ $12 \mathrm{~km}$ square. There are 20 vehicles in the area performing random walk movements defined in [28]. Velocities, directions and transition intervals follow uniform distributions with the ranges of $[10,25](\mathrm{m} / \mathrm{s}),[0,2 \pi)$ and $[1,10]$ (min) respectively.

The commander is located $1 \mathrm{~km}$ away from the border. UAVs depart from the commander, fly to the area, then perform surveillance. Starting waypoints are randomly selected to eliminate their effects on results. The endurance of UAVs is $2 \mathrm{~h}$. When the rest endurance is just sufficient to return, UAVs will return to the commander. Simulation settings are shown in Fig. 9.

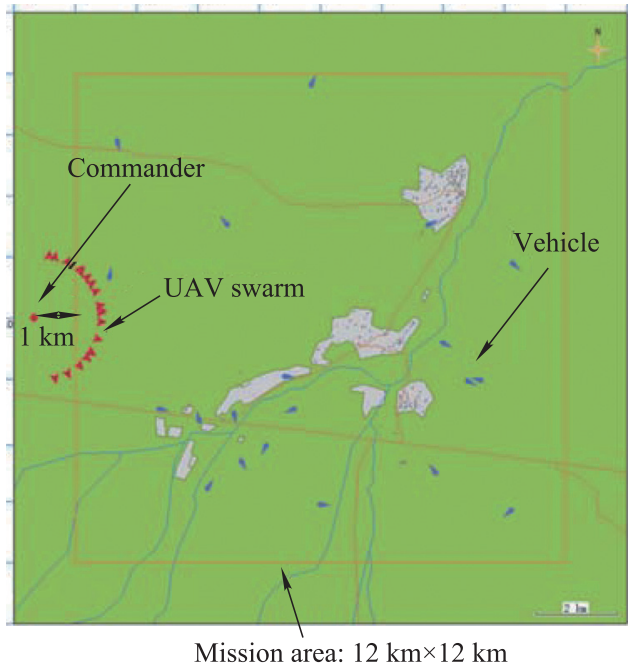

Fig. 9 Simulation settings

The simulation is programmed on the anylogic simulation platform. The platform provides basic simulation engines, such as the engines of dynamic equations, state machines, message passing, etc., while detailed models are programmed by users themselves in java. The interface of the program is shown in Fig. 10. Parameters can be manually set or automatically read from files before running the simulation.

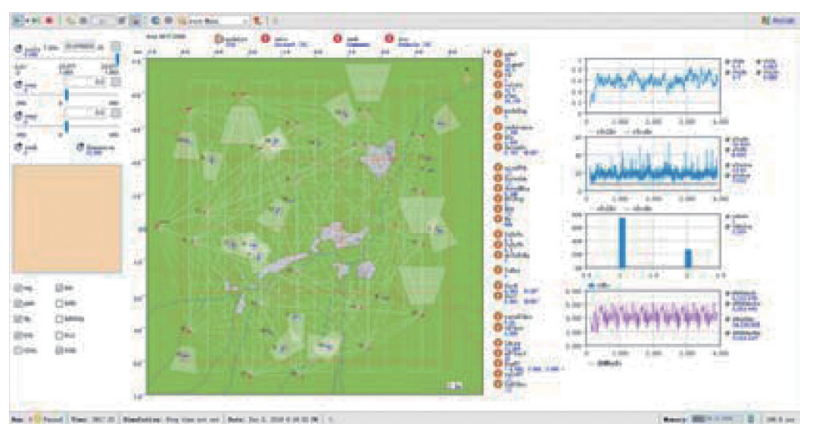

Fig. 10 Interface of simulation program

The interface can demonstrate real time agent behaviours and MoE values during the simulation. After the simulation, MoE values can be recorded in files.

The subsystems performances for trade-offs are described in Section 2.4. The bounds of communication ranges are shown in Table 1 , which are calculated by $1 \times 10^{6}$ Monte Carlo runs. The lower bound in grid configurations is significantly lower than that in vector configurations due to more uniform distributions of UAVs. The 
ranges of subsystems performances are shown in Table 2. The range of the number of UAVs $n_{\mathrm{a}}$ is [10,30]. Other subsystems and operation parameters are shown in Table 3.

Table 1 Bounds of communication range

\begin{tabular}{ccc}
\hline Configuration & Lower bound $/ \mathrm{km}$ & Upper bound $/ \mathrm{km}$ \\
\hline Vector & 9.36 & 14.63 \\
Grid & 5.89 & 14.66 \\
\hline
\end{tabular}

Table 2 Ranges of subsystems performances

\begin{tabular}{ccc}
\hline Variables & Minimum value & Maximum value \\
\hline Cruise velocity $v_{\mathrm{a}} /(\mathrm{m} / \mathrm{s})$ & 15 & 50 \\
Measurement error $\sigma_{\mathrm{m}} / \mathrm{m}$ & 3 & 30 \\
Communication range $r_{\mathrm{c}} / \mathrm{km}$ & 5.5 & 15.0 \\
Single-hop delay $t_{\mathrm{d}} / \mathrm{s}$ & 0 & 2 \\
\hline
\end{tabular}

Table 3 Subsystems and operation parameters

\begin{tabular}{cc}
\hline Parameters & Values \\
\hline FPA size $/ \mathrm{mm} \times \mathrm{mm}$ & $4.8 \times 3.6$ \\
Sensor resolution $/$ pixel $\times$ pixel & $1296 \times 736$ \\
Range of focal length $/[\mathrm{mm}, \mathrm{mm}]$ & {$[5,50]$} \\
Default look-down angle $/\left(^{\circ}\right)$ & 45 \\
Flight altitude $/ \mathrm{m}$ & 1000 \\
Track processing period $/ \mathrm{s}$ & 0.1 \\
Track transmission period $/ \mathrm{s}$ & 0.5 \\
\hline
\end{tabular}

The overall end-to-end delay is also calculated in simulations as an auxiliary measure to assist the analysis, which is expressed as follows:

$$
t_{\mathrm{deo}}=\sum_{i=1}^{n_{\mathrm{m}}} t_{\mathrm{de}, i}
$$

where $t_{\mathrm{deo}}$ is the end-to-end delay of the $i$ th message, which is defined as the difference between the time when the message is sent out from the UAV initially generating the track and the time when the message is received by the commander; $n_{\mathrm{m}}$ is the total number of the messages transmitted in the mission.

\subsection{Single-run experiment}

Take the case in which performances are the median values of respective ranges in Table 2 , the grid configuration is used as an example, and the simulation is demonstrated in Fig. 11. In this case, the communication range does not cover the entire area, so farther UAVs communicate via multi-hop links.

The variation of MoEs over time in a single run is shown in Fig. 12. When UAVs are fully deployed, the variations demonstrate that approximate cyclic patterns as surveillance paths are cyclic. The largest overall tracking ratio $r_{\text {tro }}$ reaches 0.9 , while the lowest reaches 0.3 , indicating that at the maximum $90 \%$ and at the minimum $30 \%$ of targets are monitored at any time. The overall tracking error $e_{\text {tro }}$ remains between $10 \mathrm{~m}$ to $30 \mathrm{~m}$ at most time. Sometimes $e_{\text {tro }}$ rises over $40 \mathrm{~m}$, which happens when messages need more hops to the commander.

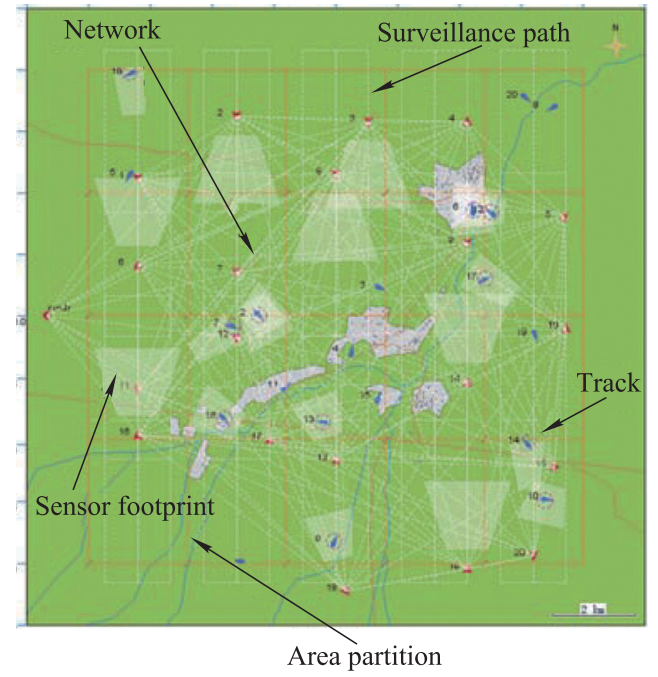

Fig. 11 Demonstration of simulation

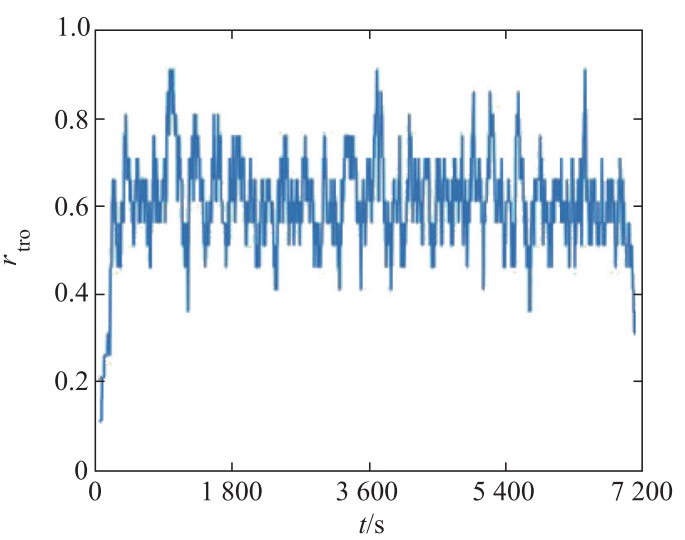

(a) Overall tracking ratio

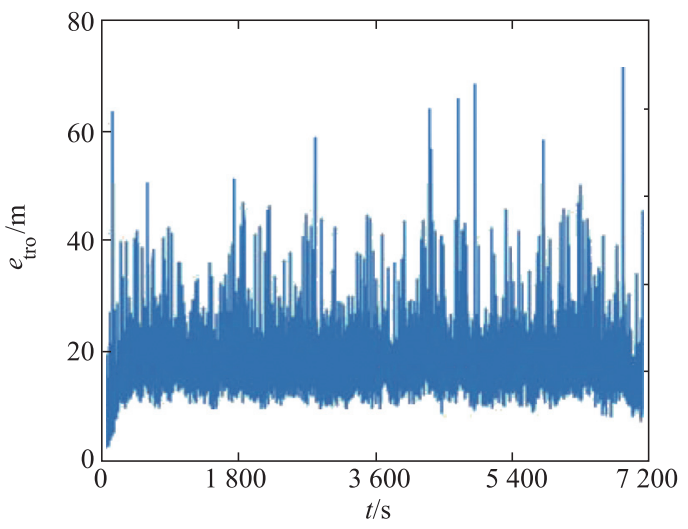

(b) Overall tracking error

Fig. 12 Variation of MoEs over time

\subsection{Rules of simulation replications}

The simulation is required to be replicated for estimating the means of MoEs as the model is stochastic. The mini- 
mum number of replications $n_{\mathrm{s}}$ is determined by two rules based on precision. In the absolute-error rule, the minimum $n_{\mathrm{s}}[29]$ is given by

$$
l_{\mathrm{hCI}}\left(n_{\mathrm{s}}\right)=t_{n-1,1-\frac{\alpha}{2}} \sqrt{\frac{s\left(n_{\mathrm{s}}\right)^{2}}{n_{\mathrm{s}}}} \leqslant \beta
$$

where $\beta$ is the required absolute error, $l_{\mathrm{hCI}}\left(n_{\mathrm{s}}\right)$ is the halflength confidence interval (CI) of the mean estimate, $s\left(n_{s}\right)$ is the sample standard deviation, and $t_{n-1,1-\frac{\alpha}{2}}$ is the upper $1-\frac{\alpha}{2}$ critical point for the $t$ distribution with $n-1$ degrees of freedom. In the relative-error rule, the minimum $n_{\mathrm{S}}$ [29] is given by

$$
r_{\mathrm{hCI}}\left(n_{\mathrm{s}}\right)=\frac{t_{n-1,1-\frac{\alpha}{2}} \sqrt{\frac{s\left(n_{\mathrm{s}}\right)^{2}}{n_{\mathrm{s}}}}}{\bar{m}\left(n_{\mathrm{s}}\right)} \leqslant \frac{\gamma}{1+\gamma}
$$

where $\gamma$ is the required relative error, $r_{\mathrm{hCI}}\left(n_{\mathrm{S}}\right)$ is the relative half-length $\mathrm{CI}$, and $\bar{m}$ is the sample mean. Besides, the central limit theorem requires $n_{\mathrm{s}}$ more than 40 . Details are described in [29]. The absolute-error rule is applied to the overall tracking ratio because the range of the ratio is close to 0 , while the relative-error rule is applied to the overall tracking error and the overall end-to-end delay because the values are case-dependent.

Frequency distribution histograms of MoEs with 500 runs are shown in Fig. 13. The distributions approximate the normal distribution. The variations of half-length CIs of $r_{\text {tro }}$ and relative half-length CIs of $e_{\text {tro }}$ with $n_{\mathrm{s}}$ are shown in Fig. 14. The drop rates of CIs decrease with increasing $n_{\mathrm{s}}$, and thus the required error should not be set excessively low considering the computation cost. We set the required errors of $e_{\text {tro }}, r_{\text {tro }}$ and $t_{\text {deo }}$ to be $0.005,0.5 \%$, and $0.01 \mathrm{~s}$, respectively.

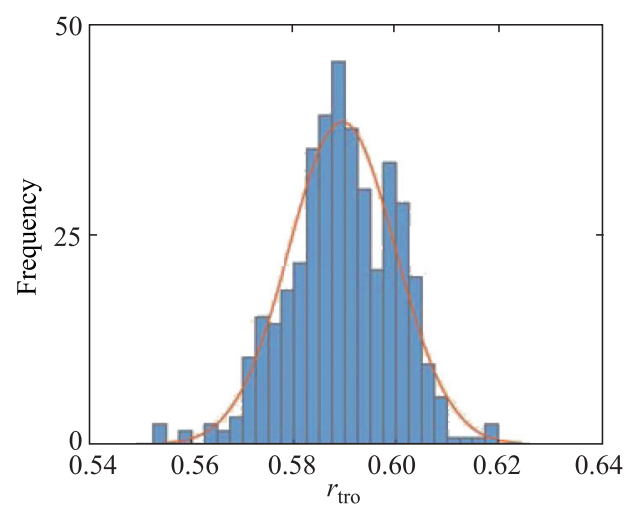

(a) Overall tracking ratio

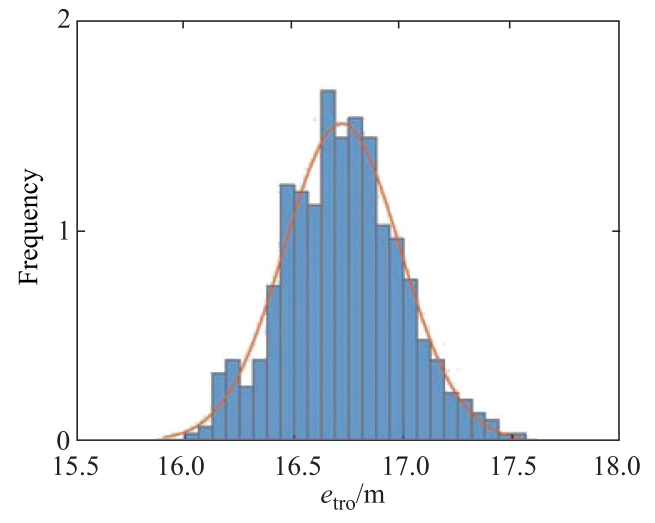

(b) Overall tracking error

Fig. 13 Frequency distribution histograms of MoEs

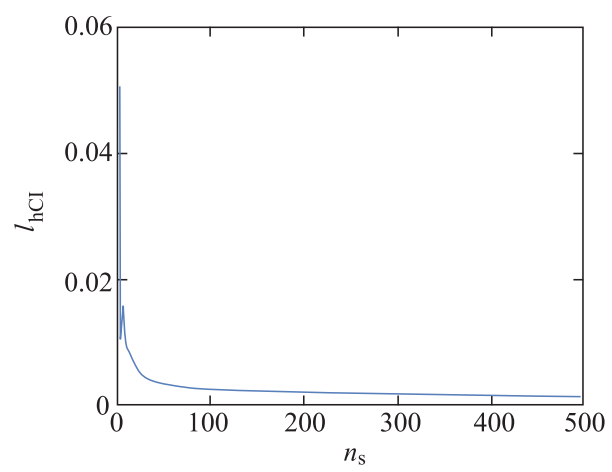

(a) Overall tracking ratio

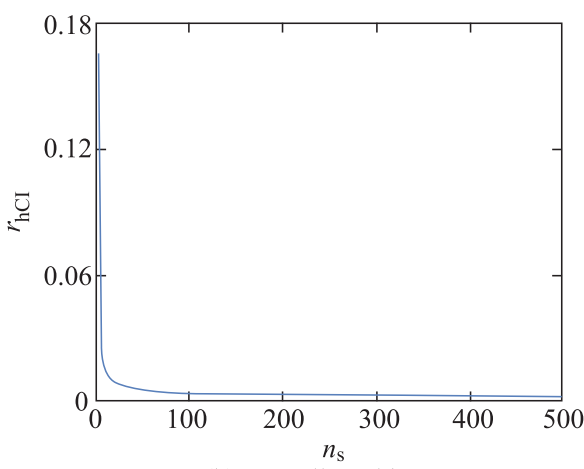

(b) Overall tracking error

Fig. 14 Variation of CI with the number of runs

\subsection{Trade-off experiments}

We set two separate experiments in trade-offs: one uses vector configurations, and the other uses grid configurations. In each experiment, the number of UAVs $n_{\mathrm{a}}$ and the subsystem performances are taken as factors.

The second-order response surface surrogate models are created for the three measures in the two experiments respectively to improve the efficiency, which are expressed as follows [30]:

$$
\widehat{y}=\beta_{0}+\sum_{i=1}^{n_{v}} \beta_{i} x_{i}^{\prime}+\sum_{i=1}^{n_{v}} \sum_{j=1}^{j \leqslant i} \beta_{i j} x_{i}^{\prime} x_{j}^{\prime}
$$


where $\widehat{y}$ is the estimated response, namely one of the three measures; $x_{i}^{\prime}$ denotes the normalized variables of factors; $n_{v}$ is the number of variables; $\beta_{0}, \beta_{i}$ and $\beta_{i j}$ denote coefficients. The normalization of variables is given by

$$
x^{\prime}=\frac{x-x_{\min }}{x_{\max }-x_{\min }}
$$

where $x_{\min }$ and $x_{\max }$ are the minimum and maximum values of variables, respectively.

Samples of normalized variables are created by the optimal Latin hypercube sampling method [31]. The samples are identical for the two experiments, and the number is 50 for each. The scatter plots of samples are shown in Fig. 15, showing that the uniformity is satisfactory.

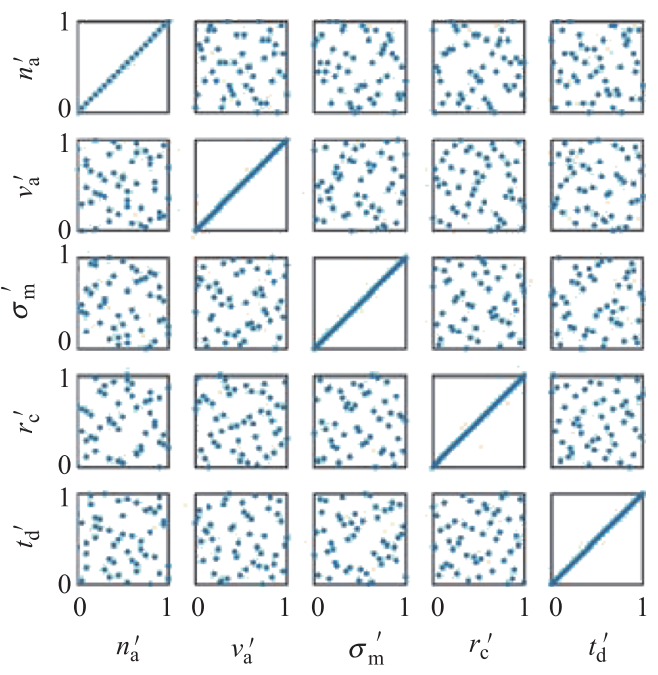

Fig. 15 Scatter plots of samples

The simulation of each sample is conducted following the rules in Section 4.3. The average number of replications is 66 , and the maximum is 282 . The root-mean-square errors (RMSEs) of the surrogate models are shown in Table 4, and the scatter plots of estimated responses of surrogate models versus real responses of samples are shown in Fig. 16, showing that the accuracy is satisfactory.

Table 4 RMSEs of surrogate models

\begin{tabular}{ccc}
\hline Partitioning configuration & Vector & Grid \\
\hline Overall tracking ratio $r_{\text {tro }}$ & 0.014 & 0.005 \\
Overall tracking error $e_{\text {tro }} / \mathrm{m}$ & 0.62 & 0.56 \\
Overall end-to-end delay $t_{\text {deo }} / \mathrm{s}$ & 0.057 & 0.048 \\
\hline
\end{tabular}

By using the surrogate models, we first analyze main affecting factors by the factorial design. Five levels are set for each factor, and results are analyzed by effect plots. Then we conduct the detailed trade-offs by contour plots.
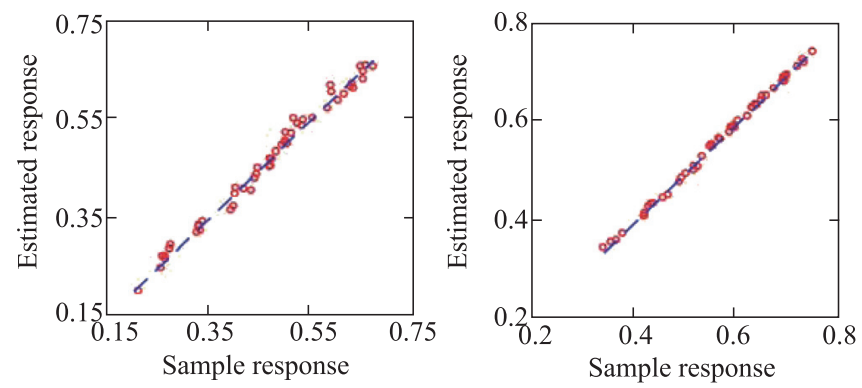

(a) $r_{\text {tro }}$, vector configuration

(b) $r_{\text {tro }}$, grid configuration

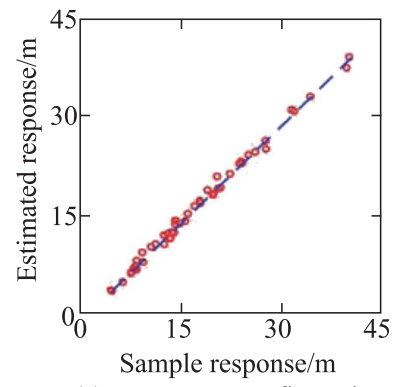

(c) $e_{\text {tro }}$, vector configuration

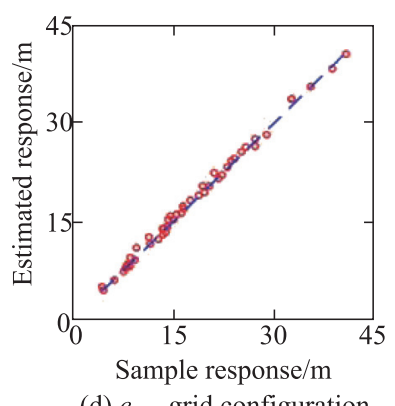

(d) $e_{\text {tro }}$, grid configuration
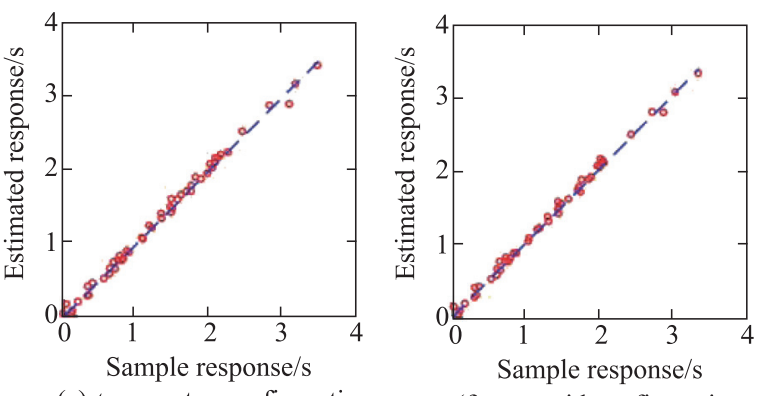

(f) $t_{\text {deo }}$, grid configuration

Fig. 16 Precision of responses surfaces

The effect plots of overall tracking ratios $r_{\text {tro }}$ in the vector configuration and the grid configuration are shown in Fig. 17(a) and Fig. 17(b) respectively. The plots are produced by a 5-level factorial design of experiments. Here we denote the variable $n_{\mathrm{a}}, v_{\mathrm{a}}, \sigma_{\mathrm{m}}, r_{\mathrm{c}}, t_{\mathrm{d}}$ as $x_{1}$ to $x_{5}$ respectively. Five levels are set for each variable, and the value of the $j$ th level of the $i$ th variable is

$$
x_{i j}=\frac{j-1}{4}\left(x_{i, \max }-x_{i, \min }\right)+x_{i, \min }, j=1,2,3,4,5
$$

where $x_{i, \min }$ and $x_{i, \max }$ are the minimum and maximum values of variables respectively, as defined in Section 4.1. A design point $\boldsymbol{D}$ is defined as a combination of the five variables, denoted by

$$
\boldsymbol{D}=\left(x_{1}, x_{2}, x_{3}, x_{4}, x_{5}\right)
$$

This way $5^{5}$ design points can be generated in total, and the value of $r_{\text {tro }}$ for each design point is calculated by using the surrogate models created above. 

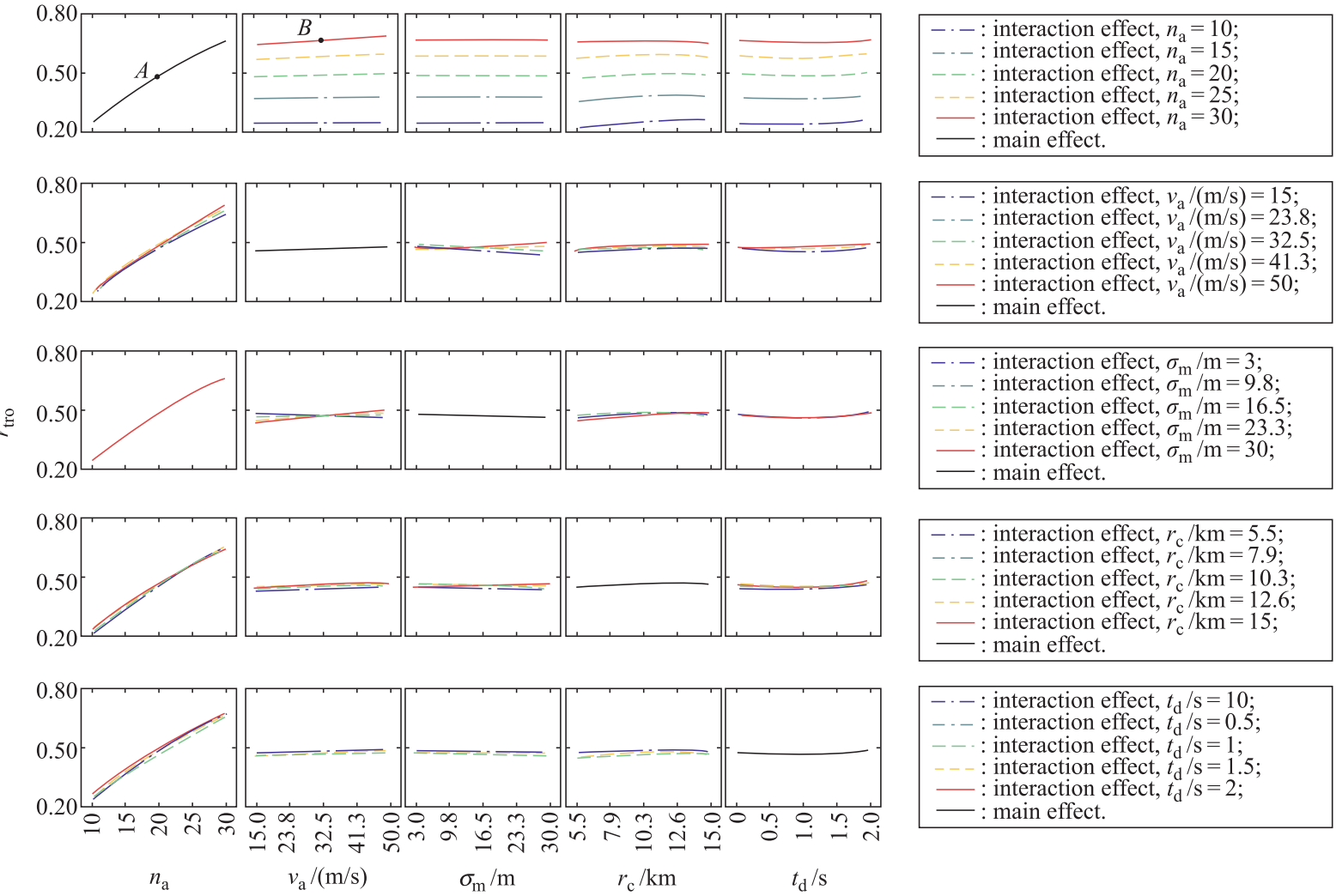

— : main effect.

$-\cdot-$ : interaction effect, $v_{\mathrm{a}} /(\mathrm{m} / \mathrm{s})=15$

: interaction effect, $v_{\mathrm{a}} /(\mathrm{m} / \mathrm{s})=23.8$;

: interaction effect, $v_{\mathrm{a}} /(\mathrm{m} / \mathrm{s})=32.5$

interaction effect, $v_{\mathrm{a}} /(\mathrm{m} / \mathrm{s})=41.3$

: interaction effect, $v_{\mathrm{a}} /(\mathrm{m} / \mathrm{s})=50$

- : main effect.

$-\cdots$ : interaction effect, $\sigma_{\mathrm{m}} / \mathrm{m}=3$

: interaction effect, $\sigma_{\mathrm{m}} / \mathrm{m}=9.8$

: interaction effect, $\sigma_{\mathrm{m}} / \mathrm{m}=16.5$;

interaction effect, $\sigma_{\mathrm{m}} / \mathrm{m}=23.3$;

: interaction effect, $\sigma_{\mathrm{m}} / \mathrm{m}=30$ : main effect.

$-\cdot-$ : interaction effect, $r_{\mathrm{c}} / \mathrm{km}=5.5$

$-\ldots$ : interaction effect, $r_{\mathrm{c}} / \mathrm{km}=7.9$;

: interaction effect, $r \mathrm{c} / \mathrm{km}=10.3$

interaction effect, $r_{\mathrm{c}} / \mathrm{km}=12.6$;

interaction effect, $r_{\mathrm{c}} / \mathrm{km}=15$

- : main effect.

$-\cdot-$ : interaction effect, $t_{\mathrm{d}} / \mathrm{s}=10$;

--- : interaction effect, $t_{\mathrm{d}} / \mathrm{s}=0.5$

interaction effect, $t_{\mathrm{d}} / \mathrm{s}=1$;

interaction effect, $t_{\mathrm{d}} / \mathrm{s}=1.5$

: interaction effect, $t_{\mathrm{d}} / \mathrm{s}=1.5$
interaction effect, $t_{\mathrm{d}} / \mathrm{s}=2 ;$

- : main effect.

(a) Vector configuration
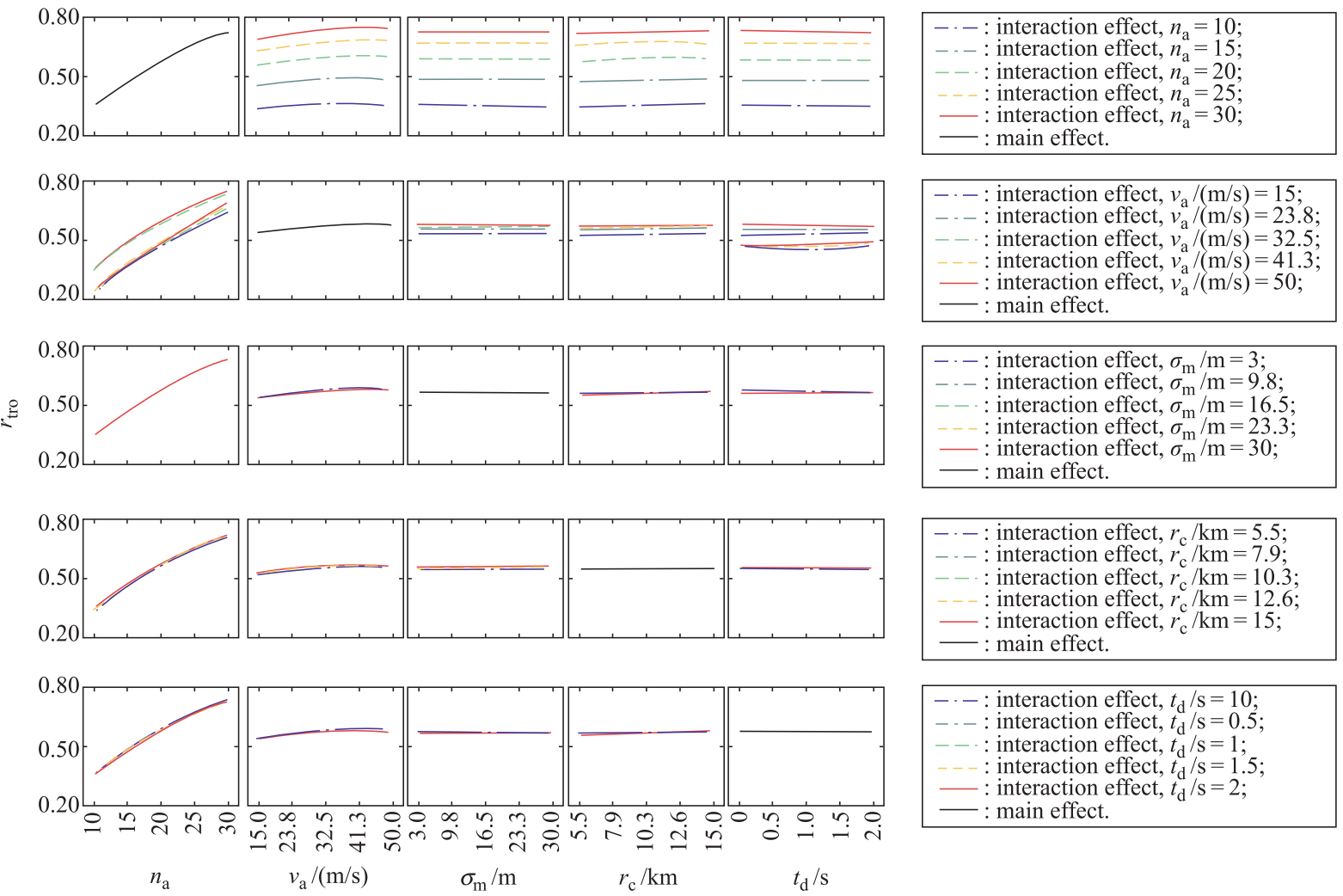

(b) Grid configuration

Fig. 17 Effect plots of overall tracking ratios 
In Fig. 17, diagonal plots are the main effects of the variables indicated at the bottom. In the plot of the $i$ th row, the $y$-axis values of the points indicate the average $r_{\text {tro }}$ of all the design points $D$ in which the $x_{i}$ equals the corresponding $x$-axis value. For example, in Fig. 17(a), the point A indicates that the average $r_{\text {tro }}$ of all the design points $D$ in which $n_{\mathrm{a}}=20$ is 0.49 . The nondiagonal plots are the interaction effects of the variable indicated at the bottom and the variable indicated by the legend on the right. In the plot of the $i$ th row and $j$ th column, the $y$-axis values of the points indicate the average $r_{\text {tro }}$ of all the design points $D$ in which the $x_{i}$ equals the corresponding $x$-axis value and the $x_{j}$ equals the value indicated by the corresponding line type. For example, the point $\mathrm{B}$ indicates that the average $r_{\text {tro }}$ of all the design points $\boldsymbol{D}$ in which $v_{\mathrm{a}}=32.5 \mathrm{~m} / \mathrm{s}$ and $n_{\mathrm{a}}=30$ is $0.67 . r_{\text {tro }}$ is mainly affected by the number of UAVs $n_{\mathrm{a}}$, the cruise velocity $v_{\mathrm{a}}$, and the communication rage $r_{\mathrm{c}}$ positively, and the effect of $n_{\mathrm{a}}$ is much more significant. $r_{\text {tro }}$ in grid configurations is higher than that in vector configurations by 0.11 at the maximum. The effect of $r_{\mathrm{c}}$ is more prominent when $r_{\mathrm{c}}$ is small in vector configurations, as the lower bounds of $r_{\mathrm{c}}$ in vector configurations are larger.

The effect plots of overall tracking errors $e_{\text {tro }}$ are shown in Fig. 18. $e_{\text {tro }}$ is mainly affected by the number of UAVs $n_{\mathrm{a}}$ and the communication range $r_{\mathrm{c}}$ negatively while affected by the measurement error $\sigma_{\mathrm{m}}$ and the communi- cation delay $t_{\mathrm{d}}$ positively. The effects of $t_{\mathrm{d}}, \sigma_{\mathrm{m}}, r_{\mathrm{c}}$ and $n_{\mathrm{a}}$ recede in order. $e_{\text {tro }}$ in vector configurations is slightly lower by $2.0 \mathrm{~m}$ at the maximum. The effect plots of overall end-to-end delays $t_{\text {deo }}$ are shown in Fig. 19. It should be noticed that $t_{\text {deo }}$ is not affected by $n_{\mathrm{a}}$, and $t_{\mathrm{deo}}$ in vector configurations is slightly higher by $0.16 \mathrm{~s}$ at the maximum. Therefore, the decrease of $e_{\text {tro }}$ with increasing $n_{\mathrm{a}}$ is caused by increasing sources in track fusion, and the lower $e_{\text {tro }}$ in vector configurations results from more overlaps of sensor footprints induced by less uniform distribution of UAVs.

The contour plots of MoEs with respective main affecting factors are shown in Fig. 20. The values of the other factors are median values of respective ranges in Table 2. Each contour represents a series of samples where the denoted values of MoEs occur.

As the effect of the cruise velocity $v_{\mathrm{a}}$ on both MoEs is not prominent except when $v_{\mathrm{a}}$ is low, a medium value about $35 \mathrm{~m} / \mathrm{s}$ is sufficient. For the trade-off among the measurement error $\sigma_{\mathrm{m}}$, the communication range $r_{\mathrm{c}}$ and the single-hop delay $t_{\mathrm{d}}$, the requirement of the overall tracking error $e_{\text {tro }}$ should be primarily considered. Considering the effect degrees of the three performances, to achieve a required level of $e_{\mathrm{tro}}$, a lower $t_{\mathrm{d}}$, a lower $\sigma_{\mathrm{m}}$ and a longer $r_{\mathrm{c}}$ are preferred in order. On the other hand, to achieve a required level of $e_{\text {tro }}$, if one of the above three performances recede, one or both of the other two performances have to be enhanced.
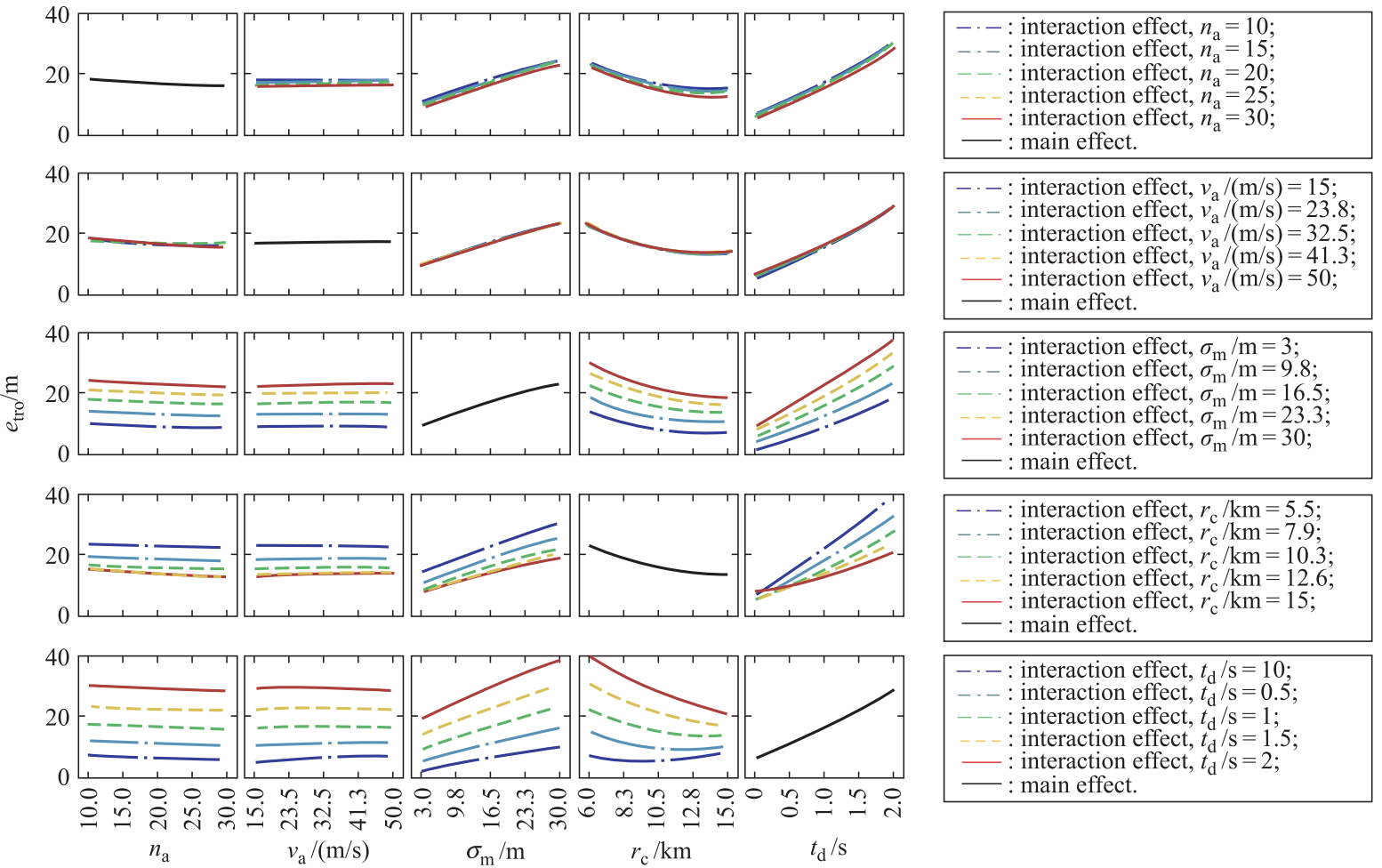

(a) Vector configuration 

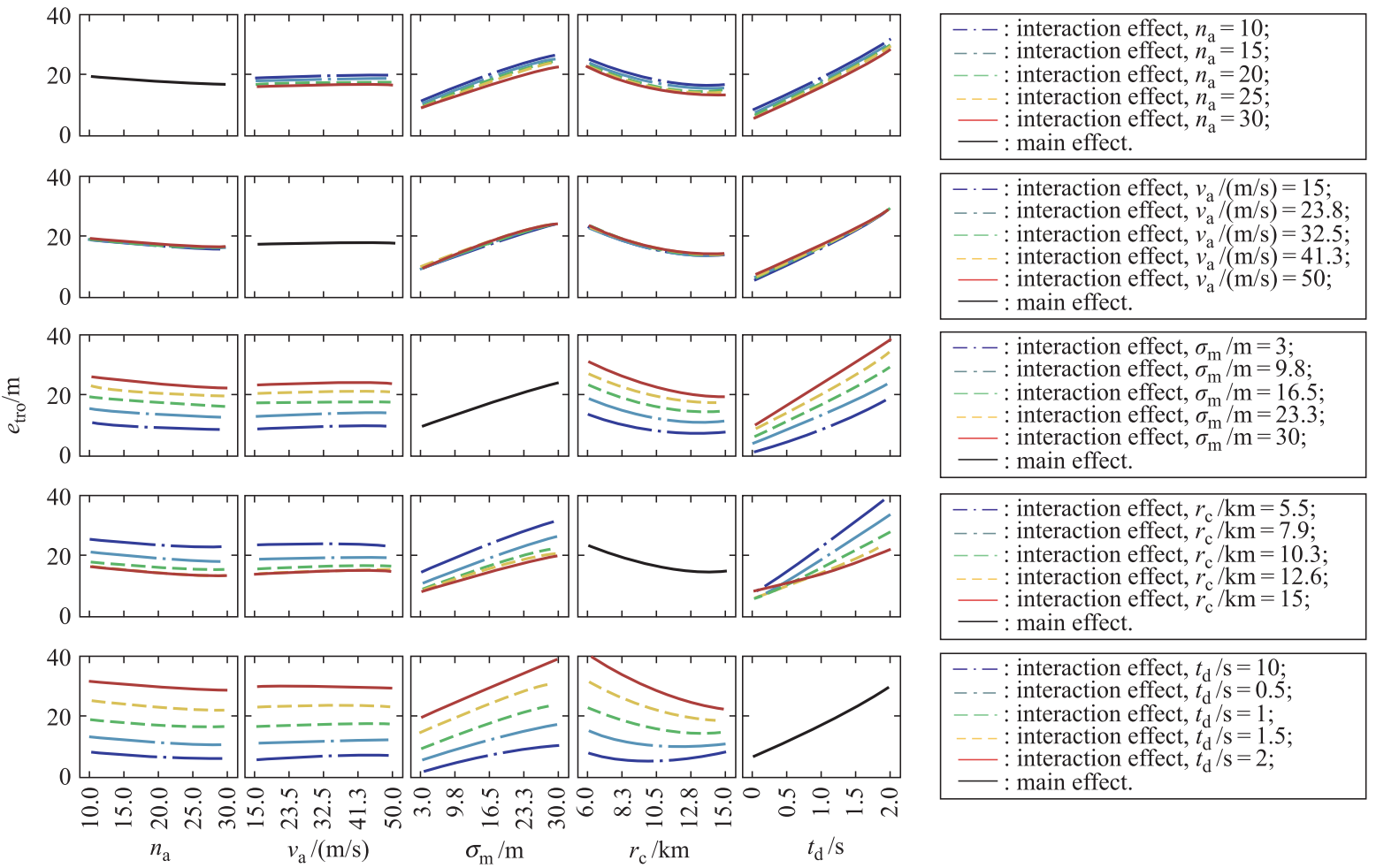

\begin{tabular}{|l|}
$-\cdot-:$ interaction effect, $t_{\mathrm{d}} / \mathrm{s}=10 ;$ \\
$--:$ interaction effect, $t_{\mathrm{d}} / \mathrm{s}=0.5$ \\
$-:$ interaction effect, $t_{\mathrm{d}} / \mathrm{s}=1 ;$ \\
$-:$ interaction effect, $t_{\mathrm{d}} / \mathrm{s}=1.5$ \\
: interaction effect, $t_{\mathrm{d}} / \mathrm{s}=2$ \\
main effect.
\end{tabular}

(b) Grid configuration

Fig. 18 Effect plots of overall tracking errors
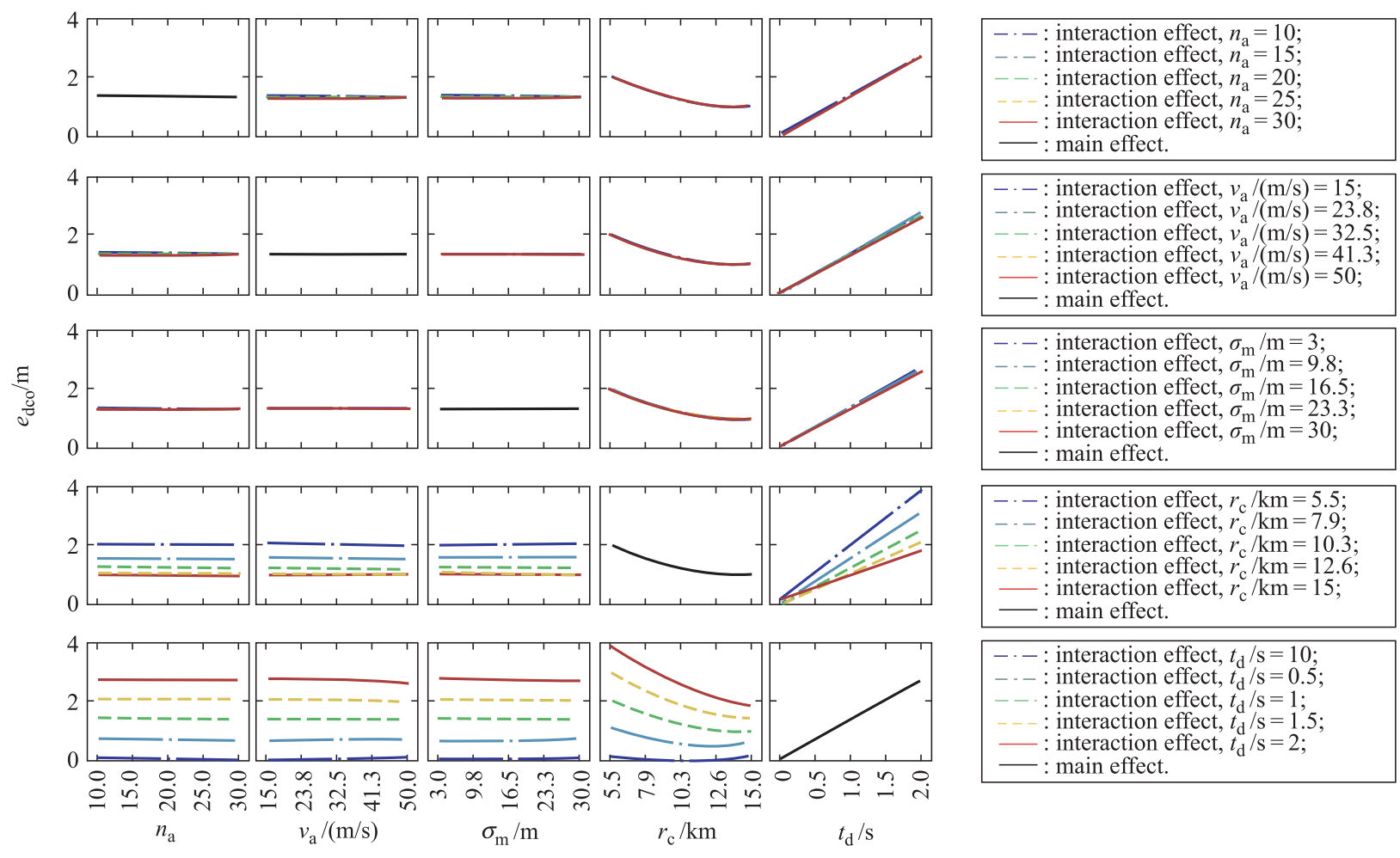

$-\cdot-$ : interaction effect, $r_{\mathrm{c}} / \mathrm{km}=5.5$

-- : interaction effect, $r_{\mathrm{c}} / \mathrm{km}=7.9$

: interaction effect, $r_{\mathrm{c}} / \mathrm{km}=10.3$.

interaction effect, $r_{\mathrm{c}} / \mathrm{km}=12.6$;

: interaction effect, $r_{\mathrm{c}} / \mathrm{km}=15$ : main effect.

$-\cdots:$ interaction effect, $t_{\mathrm{d}} / \mathrm{s}=10$
$-\cdots:$ interaction effect, $t_{\mathrm{d}} / \mathrm{s}=0.5$
$-\vdots$ interaction effect, $t_{\mathrm{d}} / \mathrm{s}=1$
$-\vdots$ interaction effect, $t_{\mathrm{d}} / \mathrm{s}=1.5$
- interaction effect, $t_{\mathrm{d}} / \mathrm{s}=2$
- main effect.

(a) Vector configuration 

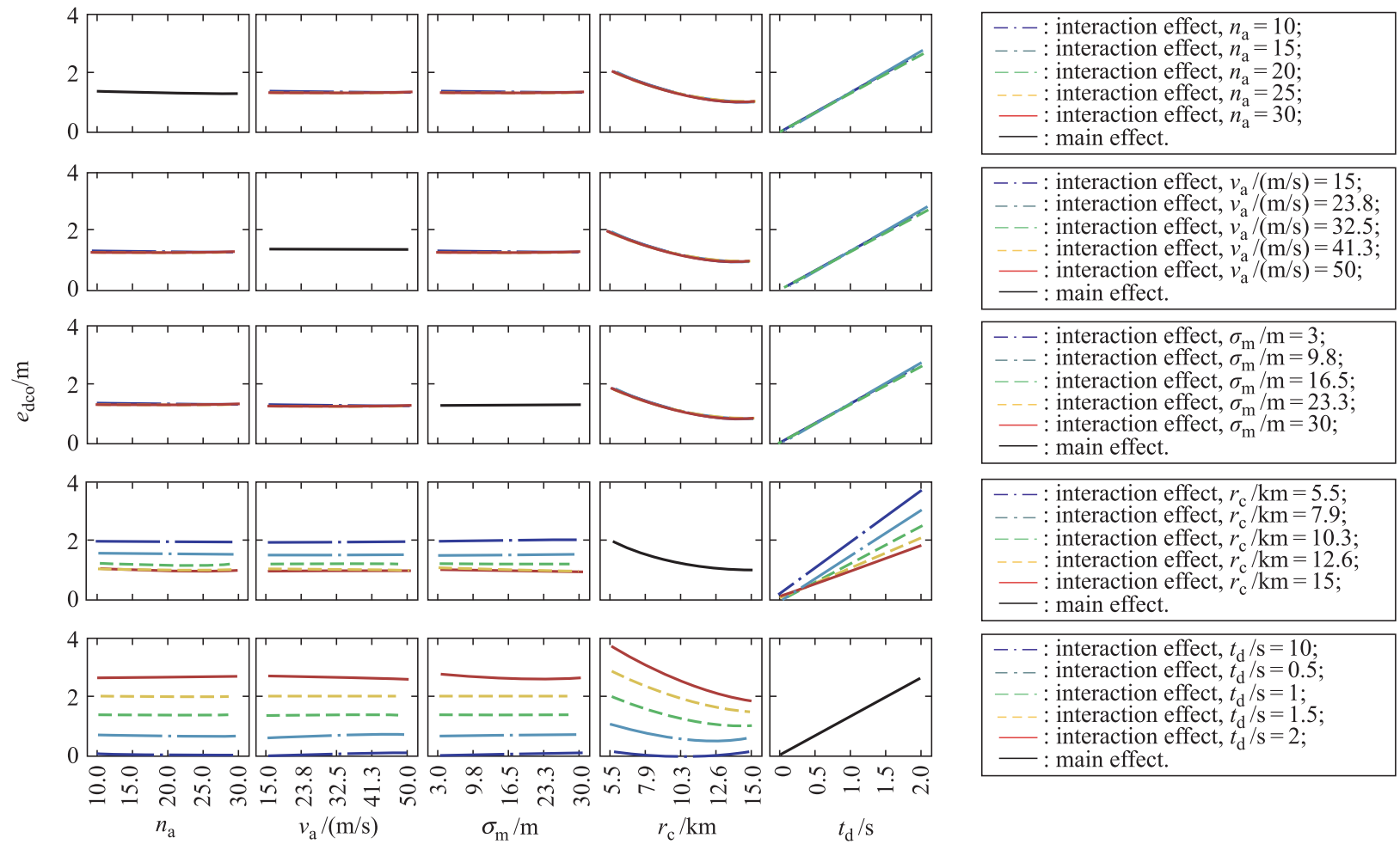

(b) Grid configuration

Fig. 19 Effect plots of overall end-to-end delays

For the choice of operational strategies, the overall tracking ratio $r_{\text {tro }}$ should be primarily considered. The grid configuration reduces the number of the needed UAVs $n_{\mathrm{a}}$ needed to achieve a required level of $r_{\text {tro }}$. However, if the requirement of $e_{\text {tro }}$ is also important, the reduction of $n_{\mathrm{a}}$ will raise the requirements of subsystems.

For example, it is required that $r_{\text {tro }}=0.5$ and $e_{\text {tro }}=$ $10 \mathrm{~m}$. From Fig. 20(a), we can find that it needs about 20 UAVs when using the vector configuration while 15 UAVs when using the grid configuration. From Fig. 19(b), if $t_{\mathrm{d}}=0.2 \mathrm{~s}$ and $r_{\mathrm{c}}=10.3 \mathrm{~km}$, it needs $\sigma_{\mathrm{m}}$ to reach $25.5 \mathrm{~m}$ when the vector configuration is used, while reach $20.8 \mathrm{~m}$ when the grid configuration is used. Therefore, when the vector configuration is used, more UAVs with lowerperformance subsystems are required, whereas when the grid configuration is used, it is the contrary.
The analyses above indicate that the overall tracking ratio $r_{\text {tro }}$ is related more to the cruise velocity $v_{\mathrm{a}}$ than to measure the error $\sigma_{\mathrm{m}}$, while the overall tracking error $e_{\text {tro }}$ is just the opposite. It can be expected that the cooperation of diverse UAVs may reduce the subsystems performance requirements. For example, the swarm can be composed of two types of UAVS: high-speed UAVs with higher $v_{\mathrm{a}}$ but higher $\sigma_{\mathrm{m}}$, and contrarily high-quality-sensing UAVs with lower $\sigma_{\mathrm{m}}$ but lower $v_{\mathrm{a}}$. High-speed UAVs search the area taking advantages of their speeds. When finding targets, they call for high-quality-sensing UAVs to track the targets instead of tracking by themselves, then continue to search the area. It makes a balance between tracking ratios and tracking errors, and reduces the requirements of partial performances in partial UAVs without decreasing the mission effectiveness.
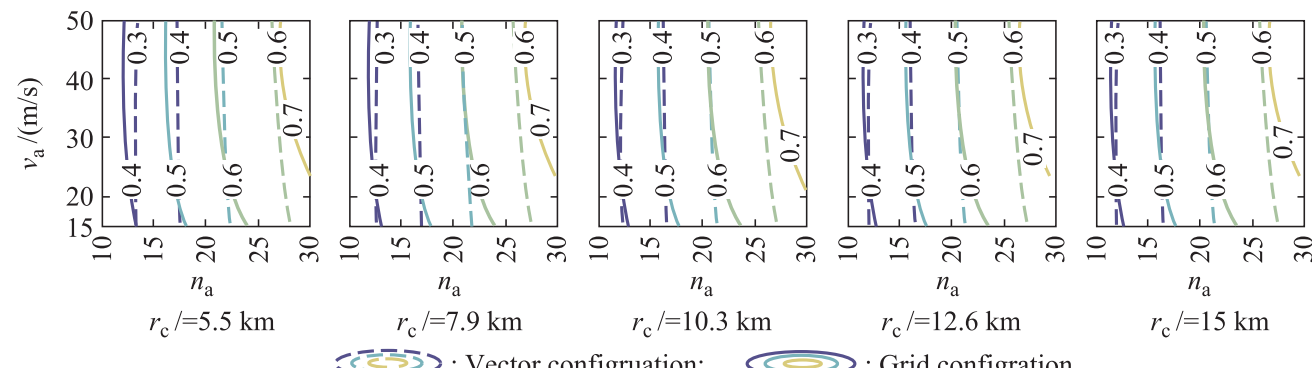

ミミンン: Vector configruation;

: Grid configration

(a) Overall tracking ratio 

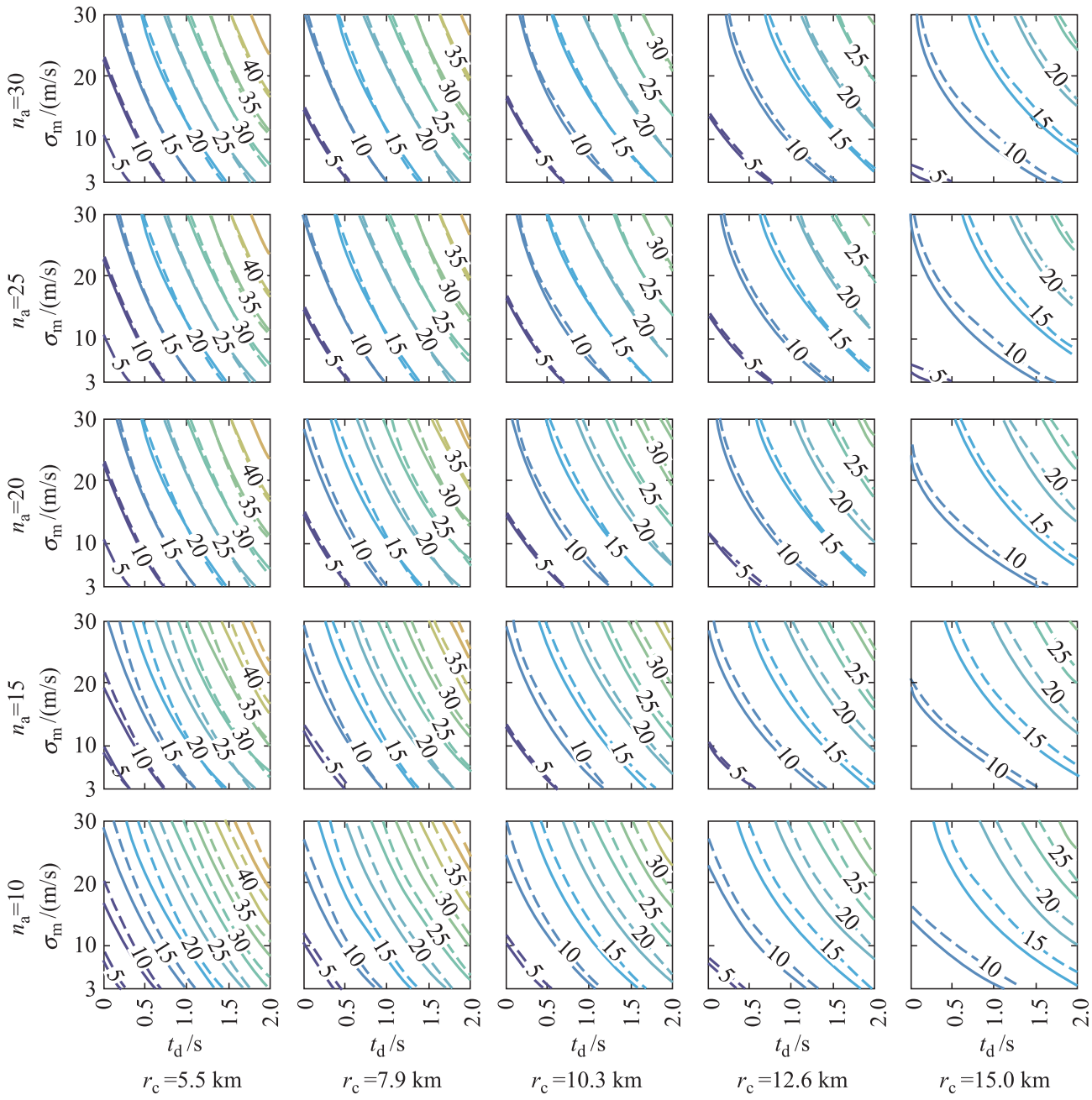

ミミミミ: Vector configruation;

$r_{\mathrm{c}}=12.6 \mathrm{~km}$

$r_{\mathrm{c}}=15.0 \mathrm{~km}$

(b) Overall tracking error

Fig. 20 Contour plots for trade-offs

However, such operations need online mission planning methods to allocate targets and plan flight paths real-time during the mission. Otherwise there will be not much difference if we just allocate diverse UAVs in different area partitions. The sweep surveillance methods studied in this paper are off-line methods predesigned before missions and cannot support such operations, while self-organized methods are more competent for that. Besides, there are more variables and more remarkable emergent pheromones in the trade-off for diverse-UAV swarms. The response surface surrogate models and factorial design cannot support such complex analyses. Improvements can be made by adding self-organized surveillance algorithms in agent models, and using better design of experiments. Those are still under development and will appear in our future works.

\section{Conclusions}

This paper proposes an improved method for the subsystems performance trade-off in the SoS oriented design of UAV swarms, specifically for surveillance missions. Within an improved design framework with subsystems disaggregation, this method is based on a behavior-based modular model structure for agent-based operational modeling and simulation, and enables the equal treatment of subsystems, the integration of operational strategies and the trade-off for track reporting.

The overall tracking ratio and the overall tracking error are proposed as measures of the effectiveness. Simulation experiments show that the overall tracking ratio is mainly affected by the number of UAVs, and that the overall tracking error is mainly affected by the sensor measurement error, the communication range and the single-hop 
delay, whose trade space is correlated. Moreover, the configuration of area partitioning changes the trade space of subsystems: the vector configuration requires the swarm with larger population but lower performance subsystems, whereas the grid configuration requires the contrary. The results indicate that operational strategies should be considered in the early phase of the conceptual design.

Future studies are suggested to add more generalizations of medium-level element classes in agent models, so as to study the trade-off and design of other elements of the SoS architectures, such as system organization and network protocols. The trade-off for the diverse-UAV swarm is also a significant issue, and further studies can be conducted by adding self-organized surveillance algorithms in agent models and using the better design of experiments. In addition, it is also suggested to integrate the performance models of subsystems, especially the correlated constraints of subsystem performances, so as to enable further tradeoffs and the entire design optimization loop.

\section{References}

[1] DURAND J, BURGAUD F, COOKSEY K D, et al. A design optimization technique for multi-robot systems. Proc. of the 55th AIAA Aerospace Sciences Meeting, 2017: 1-13.

[2] LI Q, YAN J, ZHU J, et al. State of art and development trends of top-level demonstration technology for aviation weapon equipment. Acta Aeronautica et Astronautica Sinica, 2016, 37(1): 1 - 16. (in Chinese)

[3] LIU H, TIAN Y, GAO Y, et al. System of systems oriented flight vehicle conceptual design: perspectives and progresses. Chinese Journal of Aeronautics, 2015, 28(3): 617-635.

[4] SCHUMANN B, FERRARO M, SURENDRA A, et al. Better design decisions through operational modeling during the early design phases. Journal of Aerospace Information Systems, 2014, 11(4): 195-210.

[5] TIAN Y, LIU H, HUANG J. Design space exploration in aircraft conceptual design phase based on system-of-systems simulation. International Journal of Aeronautical and Space Sciences, 2015, 16(4): 624-635.

[6] RANQUE P, FREEMAN D, KERNSTINE K, et al. Stochastic agent-based analysis of UAV mission effectiveness. Proc. of the 11th AIAA Aviation Technology, Integration, and Operations Conference, 2011: 1-15.

[7] BAI J, LI T. Evaluation of penetration mission effectiveness oriented to fighter performance parameter analysis. Acta Aeronautica et Astronautics Sinica, 2016, 37(1): 122 - 132. (in Chinese)

[8] TIAN Y, LIU H, HUANG J. Aircraft performance analysis in conceptual design phase based on system-of-systems simulations. Proc. of the International Conference on Design Engineering and Science, 2016: 1001.

[9] TRAN H T, DOMERÇANT J C, MAVRIS D N. Evaluating the agility of adaptive command and control networks from a cyber complex adaptive systems perspective. Journal of Defense Modeling and Simulation: Applications, Methodology, Technology, 2015, 12(4): 405-422.

[10] Small unmanned aircraft systems (SUAS) flight plan: $2016-$ 2036. Washington: U.S. Deputy Chief of Staff, 2016.

[11] LI X, YANG F, LEI Y, et al. A model framework-based domain-specific composable modeling method for combat sys- tem effectiveness simulation. Software and System Modeling, 2017, 16(4): $1201-1222$.

[12] GILES K, GIAMMARCO K. Mission-based architecture for swarm composability (MASC). Procedia Computer Science, 2017, 114: 57-64.

[13] SHU Z, JIA Q, LI X, et al. An OODA loop-based function network modeling and simulation evaluation method for combat system-of-systems. Proc. of the Asian Simulation Conference, 2016: $393-402$.

[14] SHU Z, WANG W, WANG R. Design of an optimized architecture for manned and unmanned combat system-of-systems: formulation and coevolutionary optimization. IEEE Access, 2018, 6: 52725-52740.

[15] LI X, CHEN Y, DONG Z. Qualitative description and quantitative optimization of tactical reconnaissance agents system organization. International Journal of Computational Intelligence Systems, 2012, 5(4): $723-734$.

[16] LI X, FU J, DONG F, et al. Formal information representation for tactical reconnaissance system organization model. Studies in Informatics and Control, 2012, 21(3): 325-331.

[17] AKSARAY D, YAZICIOĞLU A Y, FERON E, et al. Messagepassing strategy for decentralized connectivity maintenance in multiagent surveillance. Journal of Guidance, Control, and Dynamics, 2016, 39(3): $542-555$.

[18] NATO ISR tracking standard. Brussels: NATO Standardization Agency, 2014.

[19] ZHANG R, SONG B, PEI Y, et al. Agent-based analysis of multi-UAV area monitoring mission effectiveness. Proc. of the AIAA Modeling and Simulation Technologies Conference, 2017: $1-10$

[20] HE Y, XIU J, GUAN X. Radar data processing with applications. Singapore: Wiley, 2016.

[21] GUNDLACH J. Designing unmanned aircraft systems: a comprehensive approach. Reston, U.S.: AIAA, 2012.

[22] RICHARDS M A, SCHEER J A, HOLM W A. Principles of modern radar vol. I: basic principles. Edison, U.S.: SciTech Publishing, 2010.

[23] RISTIC B, FARINA A, HERNANDEZ M. Cramér-rao lower bound for tracking multiple targets. IEE Proceedings-Radar, Sonar and Navigation, 2004, 151(3): 129-134.

[24] ACEVEDO J J, ARRUE B C, MAZA I, et al. Distributed cooperation of multiple UAVs for area monitoring missions. Motion and Operation Planning of Robotic Systems: Background and Practical Approaches, 2015: 471-494.

[25] GAO C, ZHEN Z, GONG H. A self-organized search and attack algorithm for multiple unmanned aerial vehicles. Aerospace Science and Technology, 2016, 54: 229-240.

[26] LI Y, CHEN H, ER M J, et al. Coverage path planning for UAVs based on enhanced exact cellular decomposition method. Mechatronics, 2011, 21(5): 876-885.

[27] KARP B, KUNG H T. GPSR: greedy perimeter stateless routing for wireless networks. Proc. of the 6th Annual ACM/IEEE International Conference on Mobile Computing and Networking, 2000: $243-254$.

[28] GORAWSKI M, GROCHLA K. Review of mobility models for performance evaluation of wireless network. Man-Machine Interactions 3, Advances in Intelligent Systems and Computing, 2014, 242: $567-577$.

[29] LAW A M. Simulation modeling and analysis. 5th ed. New York: McGraw-Hill, 2015.

[30] MONTGOMERY D C. Design and analysis of experiments. 8th ed. Hoboken: Wiley, 2013.

[31] FORRESTER A I J, SÓBESTER A, KEANE A J. Engineering design via surrogate modelling: a practical guide. West Sussex: Wiley, 2008. 


\section{Biographies}

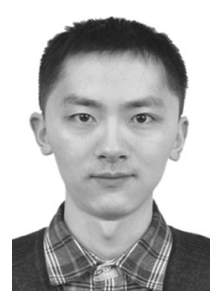

ZHANG Ruiwen was born in 1991. He received his B.S. degree in aircraft design from Northwestern Polytechnical University in 2013. Currently, he pursuits his Ph.D. degree in aircraft design at Northwestern Polytechnical University. His research interests include aircraft conceptual design, agentbased modeling and simulation, combat effectiveness analysis, and multi-agent systems.

E-mail: zhangrwchina@163.com

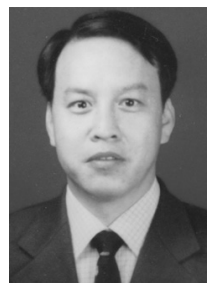

SONG Bifeng was born in 1963. He received his B.S., M.S. and Ph.D. degrees in aircraft design from Northwestern Polytechnical University in 1985, 1988 and 1998, respectively. Currently, he is a professor and doctoral supervisor at Northwestern Polytechnical University, the distinguished professor as "Chang Jiang scholars program" of Ministry of Education in aircraft design disciplines, and the chief scientist of 973 projects. His research interests include aircraft multidisciplinary design optimization and the top decision-making technology, micro aircraft design, airship design, high survivability and relia- bility technology, as well as aircraft maintainability and supportability engineering.

E-mail: bfsong@nwpu.edu.cn

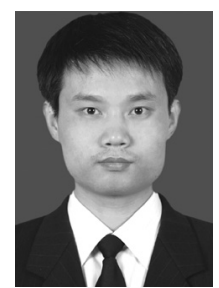

PEI Yang was born in 1978. He received his B.S., M.S., and Ph.D. degrees in aircraft design from Northwestern Polytechnical University in 2000, 2003 and 2006, respectively. Currently, he is a professor and doctoral supervisor at Northwestern Polytechnical University. His research interests include aircraft conceptual design, target damage assessment, aircraft survivability design, as well as aircraft effectiveness and cost analysis.

E-mail: peiyang_yang@nwpu.edu.cn

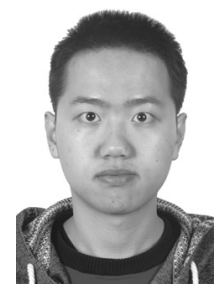

YUN Qijia was born in 1990. He received his B.S. degree in aircraft design from Northwestern Polytechnical University in 2013. Currently, He pursuits his Ph.D. degree in aircraft design at Northwestern Polytechnical University. His research interests include aircraft conceptual design, combat modeling and simulation, and combat effectiveness analysis. E-mail: 605105764@qq.com 